\title{
ROLE OF GLUTAMINE SYNTHETASE IN ANGIOGENESIS
} \section{BEYOND GLUTAMINE SYNTHESIS}

Guy Eelen ${ }^{1-3 * \$}$, Charlotte Dubois ${ }^{1,3 *}$, Anna Rita Cantelmo ${ }^{1,38}$, Jermaine Goveia ${ }^{1,3}$, Ulrike Brüning ${ }^{1,3 \$}$, Michael DeRan ${ }^{4}$, Gopala Jarugumilli ${ }^{4}$, Jos van Rijsse ${ }^{5}$, Giorgio Saladino ${ }^{6}$, Federico Comitani ${ }^{6}$, Annalisa Zecchin ${ }^{1,3}$, Susana Rocha ${ }^{7}$, Rongyuan Chen ${ }^{2}$, Hongling Huang $^{1,3 \dagger}$, Saar Vandekeere ${ }^{1,3}$, Joanna Kalucka ${ }^{1,3}$, Christian Lange ${ }^{1,3 \neq}$, Francisco MoralesRodriguez $z^{1,3}$, Bert Cruys ${ }^{1,3}$, Lucas Treps ${ }^{1,3}$, Leanne Ramer ${ }^{1,3 \#}$, Stefan Vinckier ${ }^{1,3}$, Katleen Brepoels ${ }^{1,3}$, Sabine Wyns ${ }^{1,3}$, Joris Souffreau ${ }^{1,3}$, Luc Schoonjans ${ }^{1,3}$, Wouter H. Lamers ${ }^{8}$, Yi $W^{9}$, Jurgen Haustraete ${ }^{10,11}$, Johan Hofkens ${ }^{7}$, Sandra Liekens ${ }^{12}$, Richard Cubbon ${ }^{1,3, \pi}$, Bart Ghesquière $^{13}$, Mieke Dewerchin ${ }^{1,3}$, Francesco L. Gervasio ${ }^{6,14}$, Xuri Li ${ }^{2 S}$, Jaap D. van Buu ${ }^{5}$, $\mathrm{Xu} \mathrm{Wu}^{4}$ \& Peter Carmeliet ${ }^{1-3 \&}$

(1) Center for Cancer Biology, University of Leuven, Leuven, B-3000, Belgium; (2) State Key Laboratory of Ophthalmology, Zhongsan Ophthalmic Center, Sun Yat-Sen University, Guangzhou, China; (3) Center for Cancer Biology, VIB, Leuven, B-3000, Belgium; (4) Cutaneous Biology Research Center, Massachusetts General Hospital, Harvard Medical School, Charlestown, Massachusetts 02129, USA; (5) Department of Molecular Cell Biology, Sanquin Research and Landsteiner Laboratory, Academic Medical Centre, University of Amsterdam, 1066CX Amsterdam, the Netherlands; (6) Department of Chemistry, University College London, London WC1E6BT, UK; (7) Molecular Imaging and Photonics, University of Leuven, Leuven, B-3000, Belgium; (8) Tytgat Institute for Liver and Gastrointestinal Research, Academic Medical Center University of Amsterdam, 1105BK Amsterdam, The Netherlands; (9) Center for Cell Analyses and Modelling, University of Connecticut Health Centre, Farmington 06032, USA; (10) Inflammation Research Center, VIB, Ghent, B-9000, Belgium; (11) Department of Biomedical Molecular Biology, Ghent University, Ghent, B-9000, Belgium; (12) Department of Microbiology and Immunology, University of Leuven, Leuven, B-3000, Belgium; (13) Metabolomics Core Facility, Center for Cancer Biology, VIB, Leuven, B-3000, Belgium; (14) Institute of Structural Molecular Biology, University College London, London WC1E6BT, UK. Present affiliations: (\&) Université de Lille, INSERM U1003, Physiologie Cellulaire, F-59000 Lille, France; (\$) Max-Delbrück-Center for Molecular Medicine, 13092 Berlin, Germany ; $(\dagger)$ Immunology Department, St. Jude Children's Research Hospital, Memphis 38105, USA; ( $\ddagger$ DFG-Research Center for Regenerative Therapies, Technical University Dresden, 01307 Dresden, Germany; (\#) Department of Biomedical Physiology and Kinesiology, Simon Fraser University, Burnaby V5A 1S6, Canada; (đ) Division of Cardiovascular and Diabetes Research, Multidisciplinary Cardiovascular Research Centre, University of Leeds, Leeds LS29JT, U.K. *equal contribution; ${ }^{\S} \mathrm{co}$-corresponding author

Editorial correspondence: P. Carmeliet \& G. Eelen phone: 32-16-373204 e-mail: peter.carmeliet@kuleuven.vib.be Xuri Li phone: 0086-20-87331815 e-mail: lixr6@mail.sysu.edu.cn 
Glutamine synthetase (GS) converts glutamate and $\mathrm{NH}_{4}{ }^{+}$to glutamine. GS is expressed by endothelial cells (ECs), but surprisingly shows negligible glutamine synthesizing activity at physiological glutamine levels. Nonetheless, genetic loss of GS in ECs impairs vessel sprouting during vascular development, while pharmacological GS blockade suppresses angiogenesis in ocular and inflammatory skin disease, only minimally affecting healthy adult quiescent ECs. This relies on inhibition of EC migration but not proliferation. Mechanistically, GS knockdown $\left(G S^{K D}\right)$ reduces membrane localization and activation of the GTPase RHOJ, while activating other Rho GTPases and Rho kinase (ROCK), thereby inducing actin stress fibers and impeding EC motility. ROCK inhibition rescues the $\mathrm{GS}^{\mathrm{KD}}$ EC migratory defect. Notably, GS is auto-palmitoylated and interacts with RHOJ to sustain RHOJ palmitoylation, membrane localization and activation. These findings highlight a novel molecular activity for GS, in addition to its glutamine synthesizing activity, in EC migration during pathological angiogenesis.

Endothelial cells (ECs) line the lumen of blood vessels. Emerging evidence reveals that EC metabolism controls vessel sprouting (angiogenesis) ${ }^{1-3}$. While glutamine catabolism in ECs was recently characterized ${ }^{4}$, it remains undetermined if glutamine anabolism controls angiogenesis in vivo. Glutamine is a carbon and nitrogen donor for biomolecule production and is involved in redox homeostasis. Most cells take up glutamine and thus do not need to synthesize it. Nonetheless, certain cell types express glutamine synthetase (GS; also called glutamate-ammonia ligase; GLUL), the enzyme capable of de novo glutamine production from glutamate and ammonia in an ATP and $\mathrm{Mg}^{2+} / \mathrm{Mn}^{2+}$ requiring reaction. GS serves also another biochemical function, i.e. ammonia clearance, but this is best described for hepatocytes, astrocytes and muscle. ECs also express $\mathrm{GS}^{5}$, though its role and importance in angiogenesis remain puzzling, given that ECs are exposed to high plasma glutamine levels. Global GS deficiency causes embryonic lethality, presumably 
68 due to the inability to detoxify ammonia ${ }^{6}$. GS deficiency in humans is extremely rare and

69 leads to multi-organ failure with infant death ${ }^{7}$. If and how GS affects angiogenesis has never been analyzed. Here we characterized the role and importance of GS in vessel

71 sprouting.

72

73

74

75

76

77

78

79

80

81

82

83

84

85

86

87

88

89

90

91

92

\section{VESSEL SPROUTING REQUIRES ENDOTHELIAL GS}

We checked GS expression in endothelial cells of the retinal microvasculature with a genetic GS reporter mouse $\left(G S^{+/ G F P}\right.$ mice with a nucleus-targeted GFP-lamin $A$ fusion reporter transgene in the GS ORF of one allele ${ }^{6}$ ). GFP tracing in the postnatal day 5 (P5) retinal plexus, co-stained with the endothelial cell marker Isolectin B4 (IB4; red), revealed endothelial expression of GFP (and thus of GS) in the microvasculature (Fig. 1a).

Human umbilical venous endothelial cells (further referred to as "ECs") expressed GS to similar levels as human colon ECs, liver ECs, human umbilical arterial ECs and blood outgrowth ECs (BOECs), but to a lower level than lung ECs (Extended Data Fig. 1a). However, GS expression in ECs or isolated mouse liver ECs (mLiECs) was lower than in HEPG2 hepatocellular carcinoma cells or astrocytes (Extended Data Fig. 1a-c), known to highly express GS. Glutamine withdrawal (below physiological concentration of $0.6 \mathrm{mM}$ ) increased GS protein levels in ECs (Fig. 1b; Extended Data Fig. 1b), as previously documented for other cell types ${ }^{8}$.

We intercrossed $G S^{l o x / l o x}$ mice with two different EC-specific tamoxifen inducible Cre driver lines, i.e. VE-cadherin(PAC)-Cre ${ }^{E R T 2}$ and $P d g f b-C r e^{E R T 2}$ mice to obtain respectively $\mathrm{GS}^{\mathrm{VECKO}}$ and $\mathrm{GS}^{\mathrm{pECKO}}$ mice. Correct recombination of the loxed GS allele was confirmed (Extended Data Fig. 1d-e) and caused an average $84 \%$ reduction of GS mRNA levels in mLiECs isolated from GS ${ }^{\mathrm{vECKO}}$ mice (Fig. 1c). In the neonatal retina, vascular plexi in P5 GS $^{\mathrm{vECKO}}$ mice showed hypobranching and reduced radial expansion, whereas vessel coverage by $\mathrm{NG}^{+}$pericytes and vessel regression (number of empty collagen $\mathrm{IV}^{+}$ 
sleeves) were unaffected (Fig.1d-h, Extended Data Fig. 1f,g). However, the number of filopodia at the vascular front and of distal sprouts with filopodia, both parameters of EC migration, was lower in $\mathrm{GS}^{\mathrm{VECKO}}$ pups (Fig $1 \mathrm{i}-\mathrm{j}$ ). Furthermore, the complexity of the vasculature at the utmost leading front of the plexus was decreased as determined by counting the number of branches in distal sprouts (Extended Data Fig. 1h). In contrast, quantification of $\mathrm{IB}^{+} \mathrm{EdU}^{+}$cells revealed no difference in the number of proliferating ECs (Fig. 1k-m; Extended Data Fig. 1i). Hypobranching was also observed in the dorsal dermal blood vasculature in E16.5 $\mathrm{GS}^{\mathrm{vECKO}}$ embryos (Fig. 1n-r). A similar retinal phenotype was observed in $\mathrm{GS}^{\mathrm{pECKO}}$ mice (Extended Data Fig. 1j-m). Thus, loss of endothelial GS causes vascular defects by impairing EC migration but not proliferation.

The retinal vascular defect restored over time (Extended Data Fig. 1n-u) and at 6 weeks, GS $^{\mathrm{VECKO}}$ animals (with GS deleted in ECs at P1-P3) did not show overt vascular defects (Extended Data Fig. 1v-ag). GS ${ }^{\mathrm{vECKO}}$ animals gained normal body weight, and blood biochemistry and hematological profiles were normal at 6 weeks (Extended Data Table 1). Vascular restoration may relate to the possibility that homozygous mutant ECs were outcompeted over time by residual wild type ECs, in which recombination did not occur (as documented in mice with endothelial loss of other key metabolic genes ${ }^{9}$ ) or because of other compensatory adaptations. Alternatively, the results raise the question if the effect of endothelial GS loss may be larger in growing (motile) ECs during vascular development than in quiescent (non-motile) ECs during adulthood in healthy conditions.

We then explored if pharmacological blockade of GS with methionine sulfoximine (MSO), which irreversibly blocks its catalytic activity, reduced pathological angiogenesis. First, in the oxygen-induced model of retinopathy of prematurity $(\mathrm{ROP})^{2,3}$, treatment of pups with MSO reduced the formation of pathological vascular tufts (Fig. 2a-c), while modestly increasing the vaso-obliterated area (Fig. 2d and Extended Data Fig. 1ah-ai). Second, we used the corneal micro-pocket assay (CPA) in mice with slow-release basic fibroblast 
119 growth factor (bFGF) containing pellets as a model of corneal neovascularization. 120 Inclusion of MSO in the pellet reduced formation of new CD31 ${ }^{+}$blood vessels in the 121 otherwise avascular cornea (Fig. 2e-g). Finally, we used the imiquimod-based mouse 122 model of inflammation-driven skin psoriasis and found a remarkable dose-dependent 123 reduction of the $\mathrm{CD} 105^{+} \mathrm{EC}$ area upon topical treatment of the affected skin with MSO 124 (Fig. 2h-I). Thus, pharmacological GS blockade inhibits pathological angiogenesis in the 125 inflamed skin and in several eye disorders.

\section{SiLENCING GS REDUCES EC MIGRATION}

127 We then used GS knockdown $\left(G S^{K D}\right.$ ) ECs (shRNA-mediated; >80\% silencing; Extended 128 Data Fig. 2a) in in vitro spheroid sprouting assays to assess vessel sprouting. GS ${ }^{\mathrm{KD}}$ 129 reduced the number of sprouts per spheroid and the total sprout length (Fig. 3a,b,e,f). Re130 introduction of a shRNA resistant $G S\left(\mathrm{rGS}^{\mathrm{OE}}\right)$ rescued the sprouting defect (Extended data 131 Fig. $2 b-c)$. The sprouting defect in $G S^{K D}$ spheroids was maintained upon mitotic 132 inactivation of ECs with mitomycin C (MitoC) (Fig. 3c-f), further suggesting an EC motility 133 defect. In agreement, at physiological glutamine levels, $\mathrm{GS}^{\mathrm{KD}}$ did not affect EC proliferation 134 (Fig. 3g). The sprouting defect was also not due to reduced EC viability or increased 135 oxidative stress, or to changes in energy charge, glutathione or NADPH levels, glycolysis, 136 glucose or glutamine oxidation, or oxygen consumption (Extended Data Fig. 2d-m).

$137 \mathrm{GS}^{\mathrm{KD}}$ impaired migration in scratch-wound and Boyden chamber assays, even upon MitoC 138 treatment, an effect that was rescued by re-introducing a shRNA-resistant $G S$ (rGS ${ }^{O E}$ ) 139 (Fig. 3h-i). Furthermore, sparsely seeded GS ${ }^{\mathrm{KD}}$ ECs had a reduced velocity of random 140 movement (Fig. 3j; Supplemental videos 1 and 2) and a decreased lamellipodial area (Fig. $1413 \mathrm{k}-\mathrm{m})$. Comparable results were obtained with a second non-overlapping shRNA and a 142 GS-specific siRNA (Extended Data Fig. 2a; Extended Data Fig. 3a-e). 
143 The migration defects suggested that $G S^{K D}$ perturbed the remodeling of the actin 144 cytoskeleton, necessary for cellular motility. Notably, we detected an increase in F-actin 145 levels in $\mathrm{GS}^{\mathrm{KD}}$ ECs (Fig. 3n). A role of GS in cytoskeletal remodeling was further 146 suggested by analyzing repolymerization of the actin cytoskeleton upon disruption with the 147 F-actin polymerization inhibitor latrunculin B and subsequent wash-out. Latrunculin B 148 perturbed the normal morphology of control and GS ${ }^{K D}$ ECs (Fig. 3o-r). After wash-out, 149 when control cells had rebuilt a normal actin cytoskeleton, GS ${ }^{\mathrm{KD}}$ ECs still had higher F150 actin levels, mainly originating from increased numbers of stress fiber bundles (Fig. 3s-u). $151 \mathrm{GS}^{\mathrm{KD}}$ did not alter $\alpha$-tubulin levels (Fig. 3v; Extended data Fig. 4a-h).

152 The increase in F-actin levels was also present in ECs, freshly isolated from MSO-treated 153 mice (Extended data Fig. 4i-k), and in confluent GS ${ }^{\mathrm{KD}}$ ECs aligning a scratch wound in 154 vitro (Extended data Fig. 4I-n). Confluent monolayer GS ${ }^{\mathrm{KD}}$ ECs displayed compromised 155 junctional integrity (Extended data Fig. 4o-v). Functionally, this corresponded to a 156 decrease in trans-endothelial electrical resistance (TEER) of GS ${ }^{\mathrm{KD}}$ ECs in vitro (Extended 157 data Fig. 4w) and increased leakiness of inflamed (but not healthy) vessels in vivo 158 (Extended data Fig. 4x-z).

\section{GLUTAMINE PRODUCTION BY ENDOTHELIAL GS}

160 To explore whether the migration defect was attributable to reduced de novo glutamine 161 synthesis, we measured the glutamine synthesizing activity of GS by supplementing ECs 162 with ${ }^{15} \mathrm{NH}_{4} \mathrm{Cl}$ (Extended Data Fig. 5a). At a physiological concentration of $0.6 \mathrm{mM}$ 163 glutamine or higher, the glutamine producing activity of GS was negligible, approximating 164 the level observed in ECs treated with MSO; it slightly increased only upon glutamine 165 withdrawal, presumably to compensate for the lack of available glutamine (Fig. 4a). Similar 166 results were obtained in medium containing dialyzed serum (Extended Data Fig. 5b). For 167 further details see Supplementary Discussion 1 and Extended Data Fig. 5c-n. 
168 To determine if the $G S^{K D}$ phenotype relied on the catalytic site of GS, we used previously

169 reported concentrations of $\mathrm{MSO}^{10}$, which competes with glutamate in the catalytic site of 170 GS and irreversibly blocks GS. MSO reduced EC spheroid sprouting, impaired EC 171 migration in scratch-wound assays under MitoC treatment, decreased lamellipodial area, 172 while increasing F-actin levels after latrunculin B wash-out but without affecting EC 173 proliferation (Extended Data Fig. 5o-t). Even though other (off-target) effects of 174 pharmacological GS inhibition cannot be formally excluded, MSO phenocopied the GS 175 knockdown, suggesting that the catalytic site of GS is indispensable to control EC 176 cytoskeletal homeostasis.

\section{GS INHIBITION AFFECTS RHOJ ACTIVITY}

178 Small GTPases and their effectors control F-actin levels and motility ${ }^{11}$, thus we explored if 179 Rho GTPases were downstream targets of GS. We focused on RHOJ, since it is EC180 enriched $^{12}$, and blocking endothelial RHOJ was proposed to be a novel anti-angiogenesis 181 approach ${ }^{13}$. Of note, RHOJ ${ }^{\mathrm{KD}}$ ECs fully phenocopied $\mathrm{GS}^{\mathrm{KD}}$ ECs in terms of decreased 182 mobility and barrier function (data not shown).

183 Since RHOJ localizes to plasma and organelle membranes to become activated ${ }^{14}$ and 184 RHOJ is almost exclusively detected in the membrane fraction ${ }^{15}$, we explored if GS levels 185 regulated RHOJ's membrane localization and activity. Immunoblotting revealed that RHOJ 186 was only detectable in the membrane fraction of ECs (consistent with previous findings ${ }^{15}$ ), 187 and that $G S^{K D}$ decreased the amount of RHOJ in the membrane fraction (without 188 concomitant increase in the cytosolic fraction, possibly because of proteasomal 189 degradation $^{16}$ ) as well as the levels of active RHOJ (Fig. 4b,c). GS ${ }^{K D}$ did not overtly affect 190 RHOJ transcript levels (relative mRNA levels: $0.99 \pm 0.03$ in control vs $0.85 \pm 0.05$ in $191 \mathrm{GS}^{\mathrm{KD}} ; \mathrm{n}=3, P=0.0282$ ). 
192 We also explored if $G^{K D}$ affected other Rho GTPases in ECs. We focused on the 193 RHOA/B/C - Rho kinase (ROCK) - myosin light chain (MLC) axis, as silencing of 194 endothelial RHOJ increases signaling of this pathway and induces aberrant F-actin stress 195 fiber formation through an as yet undefined mechanism ${ }^{13,17}$ (Fig. 4d). Standard GST196 Rhotekin pull-down assays showed that $\mathrm{GS}^{\mathrm{KD}}$ increased the activity of RHOA and RHOC, 197 but not of RHOB (Fig. 4e-g). Of note, GS ${ }^{K D}$, much like other stimuli, increased total RHOB 198 levels. We confirmed the increase in RHOA activity at the individual cell level with a 199 DORA-RHOA-FRET biosensor (Fig. 4h; Extended Data Fig. 6a), and observed that the 200 abnormally elevated RHOA activity in retracting lamellipodia in GS ${ }^{\mathrm{KD}}$ ECs evoked more 201 numerous, but smaller and more short-lived lamellipodia (Fig. 4i), which could contribute to 202 the motility impairment. As suggested previously ${ }^{18}$, increased RHOA activity in 203 lamellipodia locally leads to actomyosin contraction through ROCK and pMLC, thereby 204 prematurely retracting the lamellipodium. Combining $G S^{K D}$ and $R H O J^{K D}$ did not further 205 increase RHOA activity (data not shown) confirming that RHOJ silencing by itself 206 increased RHOA activity and suggesting that GS indeed primarily acts via RHOJ to control 207 RHOA signaling.

208 Downstream of Rho GTPases, GS ${ }^{K D}$ and MSO-treated ECs had elevated ROCK1 and 209 ROCK2 protein levels (Fig. 4j), and enhanced ROCK activity, as determined by pMLC 210 protein levels, which were similarly induced in $G S^{K D}$ and RHOJ ${ }^{K D}$ ECs (Fig. 4k; Extended 211 Data Fig. 6b-n). In agreement, ROCK inhibitors (Y27632, fasudil hydrochloride and H1152 212 dihydrochloride (not shown)) rescued the GS $^{\mathrm{KD}}$ phenotype (Fig. 4I-o; Extended Data Fig. 213 6o-w) whereas myosin light chain kinase (MLCK) inhibitors (ML7; peptide 18) did not 214 (Extended Data Fig. 6x-aa), suggesting that MLC phosphorylation through ROCK rather 215 than MLCK is more important in mediating the $G S^{K D}$ phenotype in ECs. Thus, GS ${ }^{K D}$ lowers 216 membrane localization and activity of RHOJ, while activating RHOA, RHOC, and ROCK. 
217 We explored with which of these Rho GTPases GS interacted, assuming that such an

218 interaction might facilitate / be necessary for their activation, nonetheless keeping in mind 219 that RHOJ can negatively regulate the activity of the RHOA/ROCK/MLC axis ${ }^{13,17}$ and 220 hence that loss of a primary interaction of GS with RHOJ could indirectly explain the 221 elevated levels of RHOA/ROCK/MLC upon GS ${ }^{\mathrm{KD}}$. First, co-immunoprecipitation (co-IP) 222 assays showed interaction between endogenous RHOJ and GS (Fig. 5a). Such co-IP was 223 not observed for RHOA and RHOC (most abundant in ECs) (Extended Data Fig. 7a). 224 Second, deletion of the first $20 \mathrm{~N}$-terminal amino acids in RHOJ ( $\Delta \mathrm{N} 20-\mathrm{RHOJ})$, mediating 225 RHOJ's plasma membrane localization ${ }^{19}$, reduced the interaction with GS (Extended data 226 Fig. 7b). Third, immunoblotting showed that only RHOJ, but not RHOA or RHOC, was 227 predominantly membrane localized (Extended data Fig. 7c). Fourth, we confirmed the GS228 RHOJ interaction with a bimolecular fluorescence complementation approach (BiFC) 229 (Extended Data Fig. 7d,e). Based on the above data, we focused on RHOJ as most likely 230 interacting partner of GS.

231 To interact with membrane-localized (active) RHOJ, GS should be membrane localized as 232 well. Indeed, cell fractionation studies revealed that a fraction of GS was membrane 233 localized (Fig. 5b). Further evidence derives from single particle tracking data, acquired by 234 photoactivated localization microscopy imaging (SPT-PALM), combined with total internal 235 reflection fluorescence microscopy (TIRF). We traced the movement of single GS proteins 236 tagged with the photoswitchable fluorescent protein (PSFP) mEOS (GS-mEOS). Single 237 GS-mEOS particles had a lower diffusion coefficient (DF) in the TIRF region (comprising 238 the plasma membrane and the immediately adjacent cytoplasm) than free mEOS,

239 indicative of an association of GS with membrane structures (Fig. 5c; Extended Data Fig. $2407 f)$.

\section{PALmitoylation OF GS AND RHOJ}


242 Membrane localization often requires post-translational palmitoylation. We thus

243 hypothesized that GS could be palmitoylated to allow plasma membrane localization and 244 interaction with RHOJ. Therefore, we performed click chemistry with biotin-azide 245 (Extended Data Fig. 7g) on lysates from HEK293 cells overexpressing GS and treated with 246 the clickable palmitoylation probes 16C-BYA or 16C-YA. Streptavidin pull-down showed 247 clear palmitoylation of GS, as both probes labeled GS. The labeling was reduced by MSO, 248 consistent with the presumed dependency of the phenotype on the enzyme's catalytic site 249 (Fig. 5d).

250 GS was anecdotally reported previously to be palmitoylated, however without further in251 depth molecular / functional characterization ${ }^{20}$. To determine if GS undergoes 252 autopalmitoylation, we incubated purified $\mathrm{GS}^{21}$ with palmitoyl-alkyne CoA (a substrate for 253 palmitoylation) in a cell-free system without any other proteins present, to demonstrate a 254 direct effect. Click chemistry revealed that increasing the dose of palmitoyl-alkyne CoA 255 resulted in increased autopalmitoylation of GS (Fig. 5e). Importantly, autopalmitoylation of 256 GS was achieved with physiological concentrations of palmitoyl-CoA $(1-10 \mu \mathrm{M})$ at neutral $257 \mathrm{pH}$, suggesting physiologically relevant autopalmitoylation and was confirmed with two 258 alternative methods (Supplementary Discussion 2 and Extended Data Fig. 7h-j).

259 Palmitoylation of target proteins by palmitoyl-acyl transferases (PATs) is a two-step 260 reaction, requiring first autopalmitoylation of the PAT, and thereafter, transfer of the 261 palmitoyl group to the target protein. We hypothesized GS to have a similar activity profile 262 (Supplementary Discussion 3) and explored if GS was involved in palmitoylation of RHOJ. 263 Even though RHOJ's cysteines at position 3 (C3) and 11 (C11) were in silico predicted to 264 be high fidelity palmitoylation sites (screened with SwissPalm ${ }^{22}$, data not shown), 265 palmitoylation of RHOJ has been poorly documented (except in a few studies ${ }^{23,24}$ ). 266 Interestingly, RHOJ's membrane localization and activity were reduced by treatment of 267 ECs with the pan-palmitoylation inhibitor 2-bromopalmitate (2BP) and by point mutating 
268 cysteines C3 and C11 (Fig. 5f; Extended Data Fig. 7k-t), providing initial evidence that

269 RHOJ can be palmitoylated in ECs. Using the palmitoylation probe 17-ODYA (Fig. 5g) or 270 an acyl-resin-assisted capture (acyl-RAC; Extended Data Fig. 7u), we found a reduction in 271 the levels of palmitoylated RHOJ upon blocking GS, consistent with a model whereby GS 272 sustains palmitoylation of RHOJ.

\section{Discussion}

275 Surprisingly, we found a glutamine synthesizing-independent activity for GS in regulating 276 EC motility, even though we cannot formally exclude a possible contribution of minimal 277 levels of glutamine production by GS to the observed phenotype. Indeed, GS regulates 278 RHOJ signaling in cell motility as shown by several lines of evidence. First, a fraction of 279 GS is present in EC membranes, where active RHOJ resides. Second, GS interacts with 280 RHOJ in ECs in co-IP experiments (though this interaction can be direct / indirect). Third, $281 \mathrm{GS}^{\mathrm{KD}}$ reduces RHOJ's palmitoylation, membrane localization and activity in ECs. Thus, 282 since RHOJ promotes EC motility ${ }^{13,17}$, the impaired migration of $\mathrm{GS}^{\mathrm{KD}} \mathrm{ECs}$ could be 283 attributed to the reduced RHOJ activity. RHOJ likely also indirectly contributes to 284 promoting EC motility through controlling the activity of the RHOA/ROCK/MLC signaling 285 pathway, known to regulate EC motility by affecting stress fiber formation ${ }^{13,17}$ (Extended 286 Data Fig. 7v; Supplementary Discussion 4).

287 Because purified GS seems capable of autopalmitoylation (a trademark of PAT enzymes), 288 and GS silencing lowers RHOJ palmitoylation, our data support a model, whereby GS first 289 autopalmitoylates itself and thereafter transfers the palmitoyl group to RHOJ, though we 290 cannot formally exclude that transfer of the palmitoyl group from GS to RHOJ occurs via 291 additional partners or even non-enzymatically. A possible model for GS palmitoylation is 
292

293

294 295

296

297

298

299

300

301 302

303

304

305

306

307

308

309

310

311

312

313

314

315

316

317 described in Supplementary Discussion 5, Extended Data Fig. 8 and Extended Data Table 2. Also, whether the GS-RHOJ partnership is exclusive or GS interacts with other players (eg other palmitoylated RhoGTPases such as RAC1, CDC42, RHOU or RHOV) to mediate this effect on EC motility, remains outstanding. In any case, RHOJ seems to be a critical target of GS, given that its silencing completely phenocopies GS inhibition in ECs.

Finally, GS is critical for EC motility / migration, contributing to the formation of new vessels in development and disease. In contrast, ECs do not migrate when they are quiescent in healthy adults, explaining why GS inhibition has no observable effects on the vasculature in healthy adult mice. This renders GS an attractive disease-restricted target for therapeutic inhibition of pathological angiogenesis. In agreement, the pharmacological GS blocker MSO reduced pathological angiogenesis in blinding eye and psoriatic skin disease (Fig. 2), which warrants further exploration of GS targeting in anti-angiogenesis.

(1)




\section{REFERENCES}

325

326

327

328

329

330

1 De Bock, K. et al. Role of PFKFB3-Driven Glycolysis in Vessel Sprouting. Cell 154, 651-663, doi:10.1016/j.cell.2013.06.037 (2013).

2 Schoors, S. et al. Fatty acid carbon is essential for dNTP synthesis in endothelial cells. Nature 520, 192-197, doi:10.1038/nature14362 (2015).

3 Schoors, S. et al. Partial and transient reduction of glycolysis by PFKFB3 blockade reduces pathological angiogenesis. Cell Metab 19, 37-48, doi:10.1016/j.cmet.2013.11.008 (2014).

$4 \quad$ Huang, $\mathrm{H}$. et al. Role of glutamine and interlinked asparagine metabolism in vessel formation. EMBO J 36, 2334-2352, doi:10.15252/embj.201695518 (2017).

5 Abcouwer, S. F., Lukascewicz, G. C., Ryan, U. S. \& Souba, W. W. Molecular regulation of lung endothelial glutamine synthetase expression. Surgery 118, 325334; discussion 335 (1995).

6 He, Y., Hakvoort, T. B., Vermeulen, J. L., Lamers, W. H. \& Van Roon, M. A. Glutamine synthetase is essential in early mouse embryogenesis. Dev Dyn 236, 1865-1875, doi:10.1002/dvdy.21185 (2007).

7 Haberle, J. et al. Congenital glutamine deficiency with glutamine synthetase mutations. N Engl J Med 353, 1926-1933, doi:10.1056/NEJMoa050456 (2005).

8 Kung, H. N., Marks, J. R. \& Chi, J. T. Glutamine synthetase is a genetic determinant of cell type-specific glutamine independence in breast epithelia. PLoS Genet 7, e1002229, doi:10.1371/journal.pgen.1002229 (2011).

9 Schoors, S. et al. Incomplete and transitory decrease of glycolysis: a new paradigm for anti-angiogenic therapy? Cell Cycle 13, 16-22, doi:10.4161/cc.27519 (2014). 
Dadsetan, S. et al. Brain alanine formation as an ammonia-scavenging pathway during hyperammonemia: effects of glutamine synthetase inhibition in rats and astrocyte-neuron co-cultures. J Cereb Blood Flow Metab 33, 1235-1241, doi:10.1038/jcbfm.2013.73 (2013).

Ridley, A. J. Rho GTPase signalling in cell migration. Curr Opin Cell Biol 36, 103112, doi:10.1016/j.ceb.2015.08.005 (2015).

Yuan, L. et al. RhoJ is an endothelial cell-restricted Rho GTPase that mediates vascular morphogenesis and is regulated by the transcription factor ERG. Blood 118, 1145-1153, doi:10.1182/blood-2010-10-315275 (2011).

Kim, C. et al. Vascular RhoJ is an effective and selective target for tumor angiogenesis and vascular disruption. Cancer Cell 25, 102-117, doi:10.1016/j.ccr.2013.12.010 (2014). doi:10.1042/BST20110702 (2011).

de Toledo, M. et al. The GTP/GDP cycling of rho GTPase TCL is an essential doi:10.1091/mbc.E03-04-0254 (2003).

Nethe, M. \& Hordijk, P. L. The role of ubiquitylation and degradation in RhoGTPase signalling. J Cell Sci 123, 4011-4018, doi:10.1242/jcs.078360 (2010).

Kaur, S. et al. RhoJ/TCL regulates endothelial motility and tube formation and modulates actomyosin contractility and focal adhesion numbers. Arterioscler Thromb Vasc Biol 31, 657-664, doi:10.1161/ATVBAHA.110.216341 (2011).

Heasman, S. J. \& Ridley, A. J. Multiple roles for RhoA during T cell transendothelial migration. Small GTPases 1, 174-179, doi:10.4161/sgtp.1.3.14724 (2010).

Ackermann, K. L., Florke, R. R., Reyes, S. S., Tader, B. R. \& Hamann, M. J. TCL/RhoJ Plasma Membrane Localization and Nucleotide Exchange Is Coordinately Regulated by Amino Acids within the N Terminus and a Distal Loop Region. J Biol Chem 291, 23604-23617, doi:10.1074/jbc.M116.750026 (2016). Sci U S A 78, 2120-2124 (1981). 
383

394

395

396

397

398

399

22 Blanc, M. et al. SwissPalm: Protein Palmitoylation database. F1000Res 4, 261, doi:10.12688/f1000research.6464.1 (2015).

23 Roberts, P. J. et al. Rho Family GTPase modification and dependence on CAAX motif-signaled posttranslational modification. J Biol Chem 283, 25150-25163, doi:10.1074/jbc.M800882200 (2008).

24 Wei, X., Song, H. \& Semenkovich, C. F. Insulin-regulated protein palmitoylation impacts endothelial cell function. Arterioscler Thromb Vasc Biol 34, 346-354, doi:10.1161/ATVBAHA.113.302848 (2014).

Supplementary Information is linked to the online version of the paper at www.nature.com/nature.

\section{ONLINE CONTENT}

Methods and associated references, and Extended Data display items are available in the online version of the paper. 


\section{ACKNOWLEDGMENTS}

401 We acknowledge Rod Levine for supplying purified bacterial GS, Ludo Van Den Bosch and Wendy Scheveneels for providing primary mouse astrocytes, Sander Trenson, Inne

403 Crèvecoeur, Sam Noppen, Lizette Van Berckelaer and Ria Van Berwaer for technical 404 assistance, Sarah-Maria Fendt, Dries Verdegem and Chris Ulens for discussions and 405 suggestions, Wesley Vermaelen, Abel Acosta Sanchez, Aleksandra Brajic, Agua Sobrino and Maaike Cockx for experimental assistance, Lena-Christin Conradi, Andreas Pircher for supplying materials and Els Wauters, Albert Wolthuis and Joris Jaekers for providing 408 tissues for EC isolations. HecBioSim and PRACE are acknowledged for allocation of computer time. JG, ARC, CD, CL, JK, FJMR, SR and SV are supported by the FWO; AZ by LE\&RN/FDRS; BC by IWT; UB by a Marie Curie-IEF Fellowship; HH by an EMBO 411 Iongterm fellowship; JDvB by a LSBR fellowship; RC by a British Heart Foundation 412 Intermediate Clinical Fellowship; and XW by the American Cancer Society RSG, NIH/NCl 413 and NIH/NIDDK. FC, GS and FLG are supported by EPSRC; XL by the State Key 414 Laboratory of Ophthalmology, Zhongshan Ophthalmic Center at Sun Yat-Sen University, and the National Natural Science Foundation of China (81330021 and 81670855). PC is supported by a Federal Government Belgium grant, long-term structural Methusalem

417 funding by the Flemish Government, a Concerted Research Activities Belgium grant, grants from the FWO, Foundation against Cancer and ERC Advanced Research Grant. 419 GE and MD received a Foundation against Cancer grant and GE received a FWO 'Krediet aan navorsers'.

\section{AUTHOR CONTRIBUTIONS}

Study concept and supervision: PC; contribution to the execution, support and analysis of experiments, and/or advice: GE, PC, XL, MD, LS; experimental design: GE, CD, ARC, JG and PC; molecular biology and in vivo experiments: GE, CD, ARC, JG, UB, AZ, HH, SVa, 
426 JK, CL, FMR, BC, LR, SVi, KB, SW, JS, LS, SL, RCh, RCu, MD; mass spectrometry: BG;

427 RHO activity assays: JvR, JDvB; GS palmitoylation: MDR, GJ, XW; molecular dynamics 428 simulations: GS, FC, FLG; BiFC and SPT: SR, JHo; data interpretation: GE, CD, ARC, JG, 429 RC, UB, CL, SR, LT, BC, MD, JHo, SL, BG, FLG, JDvB, XW and PC; providing necessary 430 materials: WHL, YW and JHa; manuscript drafting: GE and PC. All authors agreed on the 431 final version of the manuscript.

432

433 AUTHOR INFORMATION

434 Reprints and permissions information is available at www.nature.com/reprints.

435 The authors declare no competing interests.

436 Correspondence and requests for materials should be addressed to PC 437 (peter.carmeliet@kuleuven.vib.be).

438 


\section{LEGENDS TO FIGURES}

441 FIGURE 1: EC-SPECIFIC DELETION OF GS CAUSES VASCULAR DEFECTS IN VIVO

442 a, GS expression (arrowheads) in the retinal microvasculature (co-stained with isolectin B4 443 (IB4)) of five day-old (P5) chimeric $G S^{+/ G F P}$ pups (with zoom-in inset). b, GS protein levels 444 in HUVECs under different extracellular glutamine levels. c, GS mRNA levels upon 445 activation of VE-cadherin-Cre ${ }^{E R T 2}$. d-g, IB4 staining of P5 retinal vascular plexi from WT 446 (d) and $G^{\mathrm{VECKO}}$ (e) mice (with zoom-in insets, $A=$ =artery, V=vein) and quantification of 447 branch points at the front of the plexus (f) and radial expansion of the plexus ( $g$ ). $\mathbf{h}$, Vessel 448 regression (area of collagen IV (Col IV) ${ }^{+}$IB4 $4^{-}$vessel sleeves (\% of total $\mathrm{Col} \mathrm{IV}^{+}$area)) in 449 retinas from P5 WT and GS ${ }^{\vee E C K O}$ pups. i-j, Distal sprouts (i) and filopodia (j) at the retinal 450 vascular front. $\mathbf{k}-\mathbf{m}$, IB4 (gray)/EdU (cyan) double staining of P5 WT (I) and GS ${ }^{\mathrm{vECKO}}(\mathrm{m})$ 451 retinas (arrowheads in zoom-in insets denote $\mathrm{EdU}^{+} \mathrm{ECs}$ ) and quantification (k) of $\mathrm{EdU}^{+}$ 452 ECs at the front of the plexus. n-r, CD31-stained dermal dorsal blood vasculature in E16.5 453 WT $(n, o)$ and $\operatorname{GS}^{\mathrm{VECKO}}(\mathrm{p}, \mathrm{q})$ mice with boxed regions magnified in (o) and (q) and 454 quantification of number of branch points ( $r$ ). All data are mean \pm s.e.m; n-number 455 (individual experiments) is 2 (a,b); n-numbers (individual mice) for WT and GS ${ }^{\mathrm{VECKO}}$ are: 3 456 and 3 (c); 11 and 10 (f); 10 and 7 (g); 4 and 6 (h); 18 and 22 (i); 17 and 21 (j); 12 and 22 457 (k); 5 and $15(\mathrm{r})$, from $2(\mathrm{~g}, \mathrm{~h}, \mathrm{r}), 3(\mathrm{f})$ or $4(\mathrm{i}, \mathrm{j}, \mathrm{k})$ litters. ${ }^{\mathrm{NS}} \mathrm{P}>0.05,{ }^{*} P<0.05$ according to 458 Student's $t$ test (c,g,h,i,j,k,r) or mixed models R statistics (f). Exact $P$ values: (c) 0.0215 ; (f) 459 0.0141; (g) 0.0063; (h) 0.4902; (i) 0.0009; (j) 0.0484; (k) 0.3837; (r) 0.0046. Scale bars: 10 $460 \mu \mathrm{m}$ (a right), $50 \mu \mathrm{m}$ (a left), $100 \mu \mathrm{m}(\mathrm{l}, \mathrm{m}), 200 \mu \mathrm{m}$ (d,e,n,p). Gel source images: see 461 Supplemental Information Fig. 1.

462 
FIGURE 2: GS INHIBITION MITIGATES PATHOLOGICAL ANGIOGENESIS

466 a-d, Retinal flat-mounts of retinopathy of prematurity (ROP) mice treated with vehicle (a) or $46720 \mathrm{mg} \mathrm{kg}^{-1} \mathrm{~d}^{-1} \mathrm{MSO}$ (b). Quantification of vascular tuft (c) and vaso-obliterated area (d) in 468 control and MSO-treated ROP pups. e-g, Quantification (e) of CD31 ${ }^{+}$(green) neo-vessels 469 in corneal flat-mounts from mice in corneal pocket assays (CPA) with bFGF pellets 470 (demarcated by dotted white line) with vehicle (f) or MSO (g). h-I, CD105 staining of 471 untreated skin (h), IMQ-treated skin (i), IMQ + low dose MSO-treated skin (j), IMQ + high 472 dose MSO-treated skin (k), and quantification of $\mathrm{CD} 105^{+}$area (l). All data are 473 mean士s.e.m.; n-numbers (individual mice) for control and MSO-treated are: 7 and 6 (c,d), 47410 and 11 (e) from 3 litters (c,d) and 2 experiments (e). In (I) n=15 for control, n=22 for $475 \mathrm{IMQ}, \mathrm{n}=18$ for IMQ + MSO low (indicated by + ) and $\mathrm{n}=6$ animals for IMQ + MSO high 476 (indicated by ++ ) from 3 experiments. ${ }^{\mathrm{NS}} P>0.05,{ }^{*} P<0.05$ according to Student's $t$ test 477 (c,d,e) or ANOVA with Dunnett's multiple comparisons vs IMQ (I). Exact $P$ values (c) 478 0.0459; (d) 0.0145; (e) <0.0001; (I) ctrl vs IMQ: 0.0278; MSO low vs IMQ: 0.7283; MSO 479 high vs IMQ: 0.0451. bFGF: basic fibroblast growth factor; CD31: cluster of differentiation 480 31; IMQ: imiquimod; MSO: methionine sulfoximine. Scale bars: $100 \mu \mathrm{m}(\mathrm{a}, \mathrm{b}), 200 \mu \mathrm{m}(\mathrm{f}, \mathrm{g})$, $48175 \mu \mathrm{m}(\mathrm{h}-\mathrm{k})$.

482

FIGURE 3: LOSS OF GS IMPAIRS EC MIGRATION THROUGH PERTURBED ACTIN DYNAMICS

a-f, Control $(a, c)$ and $G S^{K D}(b, d) E C$ spheroids without $(a, b)$ and with mitomycin $C$ (MitoC) 485 $(c, d)$ treatment and number of sprouts per spheroid (e) and total sprout length (f). $\mathbf{g},\left[{ }^{3} \mathrm{H}\right]-$ 486 Thymidine incorporation in control and GS ${ }^{K D}$ ECs. h, Wound closure upon MitoC-treatment 487 of control and $G S^{K D}$ ECs. i, Boyden chamber migration for control, $G S^{K D}$ and $G S^{K D}+$ $488 \mathrm{rGS}^{\mathrm{OE}}$ (overexpression of a shRNA-resistant GS mutant) ECs, all under MitoC-treatment. 
489 j, Velocity of sparsely seeded control and GS ${ }^{K D}$ ECs. k-m, Phalloidin (F-actin) staining of 490 control (k) and $\mathrm{GS}^{\mathrm{KD}}$ (I) ECs (arrows and white dotted lines indicate lamellipodia) and 491 quantification of lamellipodial area (m). n-p, F-actin and G-actin levels in phalloidin (F492 actin) - DNAse I (G-actin) double-stained control and $\mathrm{GS}^{\mathrm{KD}} \mathrm{ECs}(\mathrm{n})$, and representative 493 images of phalloidin-stained control (o) and $G^{K D}$ (p) ECs. q-u, Phalloidin staining of 494 latrunculin B-treated control $(q, s)$ and $G^{K D}(r, t) E C s$ at timepoint $0(q, r)$ and at $1 \mathrm{~h}$ after 495 latrunculin wash-out $(\mathrm{s}, \mathrm{t})$ and quantification of F-actin levels after wash-out $(u)$. v, $\alpha$ 496 Tubulin levels in $\mathrm{GS}^{\mathrm{KD}}$ and control ECs. All data are mean \pm s.e.m.; n-numbers 497 (independent experiments) are: $4(\mathrm{e}, \mathrm{f}), 9(\mathrm{~g}, \mathrm{j}), 5(\mathrm{~h}), 6(\mathrm{i}, \mathrm{u}), 7(\mathrm{~m})$ and $3(\mathrm{n}, \mathrm{v}) .{ }^{\mathrm{NS}} \mathrm{P}>0.05$, $498{ }^{*} P<0.05$ according to mixed models $R$ statistics $(e, f)$, Student's $t$ test $(\mathrm{g}, \mathrm{h}, \mathrm{j}, \mathrm{m}, \mathrm{n}, \mathrm{u}, \mathrm{v})$ or 499 ANOVA with Dunnett's multiple comparison vs control (i). Exact $P$ values $(e, f) c t r l v s S^{K D}$ $500 \pm$ mitoC: $<0.0001$; (g) 0.7729; (h) 0.0283; (i) ctrl vs GS ${ }^{\mathrm{KD}}: 0.0093$; ctrl vs $\mathrm{GS}^{\mathrm{KD}}+\mathrm{rGS}^{\mathrm{OE}}$ : $501 \quad 0.5981$; (j) 0.0234; (m) 0.0352; (n) F-actin: 0.0467; G-actin: 0.584; (u) 0.0007; (v) 0.3491. $502 \mathrm{AU}$, arbitrary units. Scale bars: $100 \mu \mathrm{m}(\mathrm{a}-\mathrm{d}), 10 \mu \mathrm{m}(\mathrm{k}, \mathrm{l})$ and $20 \mu \mathrm{m}(\mathrm{o}-\mathrm{t})$.

503

504

\section{FIGURE 4: ENDOTHELIAL GS REGULATES RHOGTPASE ACTIVITY}

505 a, Effect of glutamine and MSO on glutamine-producing activity (\% enrichment in $\mathrm{m}+1$ 506 glutamine and glutamate, 30 min after adding ${ }^{15} \mathrm{NH}_{4}{ }^{+}$). b, RHOJ, NaK ATPase (membrane 507 marker) and GAPDH (cytosol marker) immunoblots in cytosolic (c) and membrane (m) 508 fractions with quantification c, Immunoblot for active and total RHOJ with quantification 509 (RHOJ ${ }^{\mathrm{KD}}$, beads only and irrelevant biotinylated peptide are negative controls) d, RHOJ's 510 pivotal yet incompletely understood (question mark) role in EC migration/stress fiber 511 formation. e-g, Immunoblots for pull-down RHOA (e), RHOB (f) and RHOC (g) activity 512 assays with quantifications. $\mathbf{h}$, Control and $G S^{K D}$ ECs expressing the DORA RHOA 513 biosensor, with quantification of whole-cell FRET startratio (mean \pm s.e.m.; control, $n=12$ 514 cells; $\mathrm{GS}^{\mathrm{KD}}, \mathrm{n}=9$ ). Look-up table (LUT; color bar) denotes relative RHOA activities 
515 (blue=low, red=high). i, Kymograph of DORA RHOA biosensor expressing ECs, showing 516 abnormally short-lived lamellipodia and increased RHOA activity in retracting lamellipodia 517 of GS $^{\mathrm{KD}}$ ECs (red arrowheads) (representative of 13 control and $\mathrm{GS}^{\mathrm{KD}}$ cells). j, ROCK1, 518 ROCK2, $\alpha$-tubulin immunoblots with quantification. $\mathbf{k}, \mathrm{pMLC}$, total MLC and $\alpha$-tubulin 519 immunoblots (quantification see Methods section). I, F-actin levels after latrunculin B 520 wash-out in ECs treated with the ROCK inhibitor Y27632. m-0, Effect of Y27632 on 521 spheroid sprouting defect $(m)$, migration defect $(n)$, and lamellipodial area (o). Values in 522 I,n,o are relative to untreated non-silenced control (dotted line). pMLC: phosphorylated 523 MLC. Scale bar is $25 \mu \mathrm{m}$ (h). All data are mean \pm s.e.m.; n-numbers (independent 524 experiments) are: 3 (a,e,f,m,n), 4 (c(MSO),h,k,l), 5 (o), 7 (j), 8 (c(GS $\left.\left.{ }^{K D}\right), g\right), 13$ (b). $525{ }^{N S} P>0.05,{ }^{*} P=0.05$, * $P<0.05$; ANOVA with Dunnett's multiple comparisons vs $4 \mathrm{mM}$ (a), 526 one sample $t$ test (b,c,e,f,g,j,k), Student's $t$ test (h,n,o), paired Student's $t$ test (I) or mixed 527 models R statistics (m). Exact $P$ values (a) (Glu) 0.6 mM vs 4 mM: 0.9903; 0.025 mM + 528 MSO vs 4 mM: 0.0968; 0.025 mM vs 4 mM: 0.1943; (Gln) 0.6 mM vs 4 mM: 0.4518; 0.025 $529 \mathrm{mM}+\mathrm{MSO}$ vs 4 mM: 0.9999; 0.025 mM vs 4 mM: 0.0143; (b) 0.0072; (c) MSO: 0.0323; 530 GS ${ }^{K D}$ : 0.0095; (e) 0.053; (f) 0.1790; (g) 0.0035; (h) 0.0055; (j) ROCK1 MSO: 0.0169; 531 ROCK1 GS ${ }^{K D}$ : 0.0138; ROCK2 MSO: 0.0381; ROCK2 GS ${ }^{K D}: 0.0802 ;(k)$ MSO: 0.0283; 532 GS ${ }^{K D}: 0.0431 ; \mathrm{RHOJ}^{\mathrm{KD}}: 0.0091$; (l) 0.0431; (m) $\mathrm{GS}^{\mathrm{KD}}$ vs ctrl: <0.0001; GS ${ }^{\mathrm{KD}}+\mathrm{Y} 27632$ vs 533 ctrl + Y27632: 0.5211; (n) 0.0181; (o) 0.0210. Gel source images: see Supplemental 534 Information Fig. 1.

FIGURE 5: GS (AUTO)-PALMITOYLATION

537 a, Co-immunoprecipitation (Co-IP) of endogenous RHOJ and GS in ECs. Upper panel: IP 538 of RHOJ; lower panel: IP for GS. b, Immunoblot for GS and RHOJ in cytosolic (c) and 539 membrane $(\mathrm{m})$ fractions in ECs with $\mathrm{NaK}$ and GAPDH as fraction markers. c, Diffusion 540 coefficient (DF, in $\mu \mathrm{m}^{2} \mathrm{~s}^{-1}$ ) of single photoswitchable fluorescent protein mEOS and mEOS- 
541 fused GS (mEOS-GS) particles in the plasma membrane region of ECs acquired by SPT-

542 PALM under TIRF illumination ( $n=41$ cells expressing mEOS and 37 expressing mEOS-

543 GS) d, GS immunoblotting after streptavidin pull-down of biotin-azide clicked lysates from

544 HEK-293T cells for the indicated palmitoylation probes. Input shows levels of GS

545 overexpression. e, Dose-effect of palmitoyl-alkyne CoA on autopalmitoylation of purified

546 GS; biotin-azide clicking and HRP-streptavidin blotting; input on Coomassie-stained gel. f,

547 Immunoblotting for RHOJ, NaK and GAPDH in membrane (m) and cytosolic (c) fractions of

548 control- and 2BP-treated ECs. g, Palmitoylation of RHOJ in GS ${ }^{\mathrm{KD}}$, MSO- and 2BP-treated

549 ECs. In-gel fluorescence for TAMRA-azide 17-ODYA (palmitoylation probe)-clicked FLAG-

$550 \mathrm{RHOJ}$ is shown (FLAG as loading control). 2BP, 2-bromopalmitate, pan-palmitoylation

551 inhibitor. All data are mean \pm s.e.m., except box and whisker (running from minimal to 552 maximal values) plots in (c); n-numbers (independent experiments) are: 2 (e), 3 (a,b,c,d,f),

$5534(\mathrm{~g}) .{ }^{\mathrm{NS}} P>0.05,{ }^{*} P<0.05$; Student's $t$ test $(\mathrm{c})$; one sample $t$ test $(\mathrm{f}, \mathrm{g})$. Exact $P$ values (c) 554 <0.0001; (f) 0.0264; (g) MSO: 0.0317; GS ${ }^{\mathrm{KD}}$ : 0.0003; 2BP: 0.0163. Gel source images: see 555 Supplemental Information Fig. 1. 


\section{METHODS}

570 Chemicals and ReAGents: The GS inhibitor L-methionine sulfoximine (MSO), mitomycin C,

571 latrunculin B, oligomycin, antimycin A, carbonyl cyanide-4-(trifluoromethoxy)

572 phenylhydrazone (FCCP), 2-bromohexadecanoic acid (2-bromopalmitic acid, 2BP), 573 tamoxifen, palmitoyl-CoA agarose and $\alpha$-ketoglutarate dehydrogenase were from Sigma574 Aldrich. 17-Octadecynoic acid (17-ODYA) was purchased from Cayman Chemical. The 575 use and/or synthesis of the other palmitoylation probes 15-hexadecynoic acid (16C-YA; a palmitate-based probe that binds a broader spectrum of proteins than 16C-BYA (here

577 below), including both PATs and PAT target proteins) and 2-bromooctadec-15-yonic acid 578 (16C-BYA; a 2-bromopalmitate-based activity-based probe that labels but also inhibits 579 palmitoyl acyltransferase (PAT) enzymes) has been described previously ${ }^{25}$. The ROCK 580 kinase inhibitor Y27632 $((1 \mathrm{R}, 4 \mathrm{r})-4-((\mathrm{R})-1$-aminoethyl)-N-(pyridin-4-

581 yl)cyclohexanecarboxamide) was from BioVision, fasudil hydrochloride and H1152 582 dihydrochloride are from Tocris. The MLCK inhibitors ML7-hydrochloride and peptide 18 583 were from Tocris. Collagen type 1 (rat tail) was obtained from Merck Millipore. [5- $\left.{ }^{3} \mathrm{H}\right]-$ 584 glucose, $\left[{ }^{3} \mathrm{H}\right]$-thymidine, $\left[\mathrm{U}-{ }^{14} \mathrm{C}\right]$-glutamine were from Perkin Elmer; $\left[6-{ }^{14} \mathrm{C}\right]$-D-glucose was 585 from ARC. $\left[\mathrm{U}_{-}{ }^{13} \mathrm{C}\right]-$ glucose, $\left[\mathrm{U}-{ }^{13} \mathrm{C}\right]$-glutamine, $\left[\mathrm{U}-{ }^{13} \mathrm{C}\right]$-glutamate and ${ }^{15} \mathrm{NH}_{4} \mathrm{Cl}$ were 586 purchased from Cambridge Isotope Laboratories. The following primary antibodies or dyes 587 were used (dilutions for staining (ST), immunoblotting (IB), immunofluorescence (IF) and 588 immunoprecipitation (IP) are given in between brackets): Griffonia simplicifolia (GS)-IB $4^{-}$ 589 Alexa 488 (ST 1:200), isolectin GS-IB 4 -Alexa 568 (ST 1:200), isolectin GS-IB ${ }_{4}-A l e x a ~ 647$ 590 (ST 1:200), phalloidin-Alexa 488 (ST 1:100), deoxyribonuclease I-Alexa 594 (ST 1:200) 591 (Molecular Probes), anti-collagen IV (2150-1470) (IF 1:400) (Bio Rad), anti-NG2 
592 Chondroitin Sulfate Proteoglycan (AB5320) (IF 1:200) (Millipore), anti-FLAG (clone M2) (IB 593 1:1,000; IP $5 \mu \mathrm{g} \mathrm{ml}^{-1}$ ), anti-GS (clone 2B12) (IB 1:1,000; IP 2-5 $\mu \mathrm{g} \mathrm{ml}^{-1}$ ), anti-RHOJ (clone 594 1E4) (IB 1:1,000; IP 2-5 $\mu \mathrm{g} \mathrm{ml}^{-1}$ ), anti-ROCK1 (HPA007567) (IB 1:1,000), anti- $\alpha$-tubulin 595 (T6199) (IB 1:1,000) (Sigma-Aldrich), anti-ß-actin (13E5) (IB 1:1,000), anti-phospho596 Myosin Light Chain 2 (IB 1:1,000; IF 1:300) and anti-Myosin Light Chain 2 (IB 1:1,000) 597 (9776), anti-Na,K-ATPase (NaK) (3010) (IB 1:1,000), anti-RHOA (67B9) (IB 1:1,000) and 598 anti-RHOC (D40E4) (IB 1:1,000) (Cell Signaling Technology), anti-CD105/endoglin 599 (AF1320) (IF 1:50), anti-VE-cadherin (AF1002) (IF 1:50) (R\&D Systems), anti-ROCK2 600 (A300-047A-T) (IB 1:500) (Imtec Diagnostics), anti-CD31 (MEC13.3) (IF 1:200), anti601 CD34-biotin (\#553732) (IF 1:25) (BD Biosciences), anti-RHOB (sc-180) (IB 1:1,000). 602 Secondary Alexa-405, $-488,-568$ or -647 conjugated antibodies (1:500) were from 603 Molecular Probes; other secondary antibodies and IgG controls were from Dako. The 604 Click-iT $^{\circledR}$ 5-ethynyl-2'-deoxyuridine (EdU) Alexa Fluor ${ }^{\circledR} 555$ Imaging Kit was from 605 Invitrogen. Purified bacterial GS was a kind gift from Rod Levine (Bethesda, MD, USA).

606 Cell culture: Human umbilical vein endothelial CELLS (HUVECS) and human umbilical 607 ARTERY ENDOTHELIAL CELLS (HUAECS) obtained under protocol S57123 (Commission 608 Medical Ethics of UZ/KU Leuven) after written consent of the donors, were isolated as 609 previously described ${ }^{1,2}$ and were routinely cultured in M199 medium (Invitrogen)

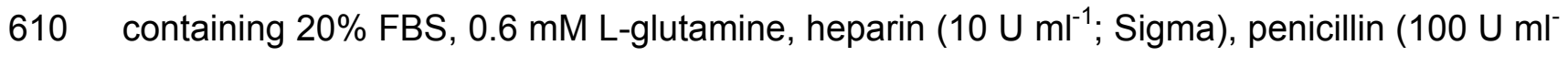

$61{ }^{1}$ ), streptomycin $\left(100 \mu \mathrm{g} \mathrm{ml}^{-1}\right)$ and endothelial cell growth factor supplements (EGCS; 30 $612 \mathrm{mg} \mathrm{l}^{-1}$; Sigma). Cells were only used between passages 1 and 4 and all experiments were 613 performed in HUVECs from at least three different donors unless stated otherwise. Also 614 except when stated otherwise, the use of the abbreviation EC in the text refers to HUVEC. 615 ISOLATION OF ENDOTHELIAL CELLS FROM HUMAN LUNG/LIVER/COLON MUCOSA: Lung/liver/colon 616 mucosa specimens were obtained under protocol S57123 (Commission Medical Ethics of 617 UZ/KU Leuven) and were washed several times with phosphate buffer solution (PBS) and 
618 minced with scissors prior to enzymatic digestion for $45 \mathrm{~min}$. at $37{ }^{\circ} \mathrm{C}$ with 619 collagenase/dispase/DNase solution (Gibco, Life Technologies). The resulting suspension 620 was passed through a $100 \mu \mathrm{m}$ nylon mesh (BD Biosciences Pharmingen) to remove 621 aggregates. The harvested cells were washed, seeded on gelatin pre-coated 6-well plates 622 and cultured in complete endothelial growth medium (EGM-MV; Lonza) supplemented with 623 antibiotics. After 5-7 days, when cells reached confluency, a positive CD31 magnetic bead 624 selection was performed (CD31 MicroBead, \#130-091-935, Miltenyi Biotech) according to 625 the manufacturer's guidelines and purified cells were further cultured in EGM medium. 626 PERIPHERAL BLOOD OUTGROWTH ENDOTHELIAL CELLS (BOECS) were established and cultured 627 as previously described ${ }^{26}$. In brief, blood samples (obtained under protocol 557123 628 (Commission Medical Ethics of UZ/KU Leuven) were diluted with PBS prior to Ficoll 629 PaquePLUS (GE Healthcare) density-gradient centrifugation at 1,000 g for 20 min at room 630 temperature. The mono-nuclear cell layer was collected, washed with PBS and 631 resuspended in EGM2 medium (PromoCell). Cells were plated in collagen-coated flasks 632 and medium was replaced every 2 days. From day 7 onwards, cells were checked for the 633 formation of colonies, which were allowed to grow up to approximately $1 \mathrm{~cm}^{2}$. BOEC 634 colonies were then trypsinized and subcultured. HEK293T AND HEPG2 CELLS (ATCC) were 635 grown in DMEM, supplemented with 10\% fetal bovine serum (FBS), $100 \mathrm{U} \mathrm{ml}^{-1}$ penicillin 636 and $100 \mathrm{\mu g} \mathrm{ml}^{-1}$ streptomycin. When HEPG2 cells were compared directly to ECs in short 637 term stable isotope tracing experiments, they were incubated in exactly the same medium 638 as the ECs to rule out possible bias coming from the difference in media formulation. We 639 did not perform authentication of the HEK293T and HEPG2 cells. MOUSE LIVER 640 ENDOTHELIAL CELLS (MLIECS) were isolated from perfused healthy livers of control or $641 \mathrm{GS}^{\mathrm{ECKO}}$ mice. Prior to perfusion, the mice were anesthetized with Nembutal $\left(60 \mathrm{mg} \mathrm{kg}^{-1}\right)$. 642 Mice were perfused with $5 \mathrm{ml}$ of a water-based perfusion buffer containing $1.7 \mathrm{M} \mathrm{NaCl}, 84$ $643 \mathrm{mM} \mathrm{KCl}, 120 \mathrm{mM}$ HEPES and $1 \mathrm{mM} \mathrm{NaOH}$ followed by $5 \mathrm{ml}$ of a PBS-based digestion 
644 buffer containing $0.1 \%$ collagenase II (Life Technologies), collagenase I (Life 645 Technologies), $2 \mathrm{mM} \mathrm{CaCl} 2,1 \%$ antibiotic-antimycotic (Life Technologies) and 10\% FBS 646 (Biochrome, Berlin, Germany) at a perfusion rate of $1 \mathrm{ml} \mathrm{min}^{-1}$. Perfusion was considered 647 complete when the liver and mesenteric vessels were blanched and the desired amount of 648 digestion buffer ( $\geq 5 \mathrm{ml}$ ) had passed through the circulatory system. Livers were dissected, 649 placed into a $50 \mathrm{ml}$ conical tube with $3 \mathrm{ml}$ of digestion buffer and incubated at $37^{\circ} \mathrm{C}$ for 650 approximately $30 \mathrm{~min}$, with regular shaking of the tubes every $5 \mathrm{~min}$. After digestion, the 651 tissue was homogeneously dissociated and the reaction was stopped with $10 \mathrm{ml}$ of 652 isolation buffer containing PBS $+0.1 \%$ BSA (Sigma-Aldrich). Subsequently, the cell 653 suspension was filtered through a $100 \mu \mathrm{m}$ cell strainer and cells were washed twice with 654 isolation buffer. Finally, the ECs were isolated by magnetic bead sorting with Dynabeads 655 (CELLection $^{\text {TM }}$ Biotin Binder Kit, Life Technologies, Ghent, Belgium) coated with anti656 mouse CD31 (eBioscience, Anti-Mouse CD31 Clone 390), according to the manufacturer's 657 instructions. Briefly, the cell suspension was incubated with the beads at room 658 temperature for 30 min in HulaMixer® Sample Mixer (Life Technologies, Ghent, Belgium). 659 Next, CD31 ${ }^{+}$ECs were collected by putting the tubes on a DynaMag ${ }^{\mathrm{TM}}-50$ Magnet (Life 660 Technologies) and removing the supernatant. The procedure was repeated twice to 661 remove cells debris. Finally, cells were resuspended in EGM2 medium (PromoCell) and 662 plated at the desired density on cell culture plates pre-coated with $0.1 \%$ gelatin, and grown 663 to confluency. MOUSE ASTROCYTES were prepared as described previously with minor 664 changes ${ }^{27}$. Briefly, spinal cords were dissected from 13-day old C57BL/6J mouse 665 embryos. Meninges and dorsal root ganglia were removed and a single cell population 666 was obtained by digestion with $0.05 \%$ trypsin in combination with gentle trituration. The 667 cell suspension was layered on a 6.2\% OptiPrep ${ }^{\mathrm{TM}}$ (Axis-Shield, Oslo, Norway) cushion 668 and centrifuged at $500 \mathrm{~g}$ for $15 \mathrm{~min}$. The pellet was resuspended and the cells were plated $669\left(12,000\right.$ cells $\left.\mathrm{cm}^{-2}\right)$ in L15 medium supplemented with glucose $\left(3.6 \mathrm{mg} \mathrm{ml}^{-1}\right)$, sodium 
670 bicarbonate $(0.2 \%)$, penicillin $\left(100 \mathrm{IU} \mathrm{ml}^{-1}\right)$, streptomycin $\left(100 \mu \mathrm{g} \mathrm{ml}^{-1}\right)$ and fetal bovine 671 serum (10\%). After reaching confluency, cell division was halted by treatment with cytosine 672 arabinoside (10 $\mu \mathrm{M}, 3$ days). After 4 weeks, more than $95 \%$ of cells stained positive for 673 glial fibrillary acidic protein (GFAP; not shown). We routinely tested primary cells and cell 674 lines for mycoplasma contamination with the MycoAlert mycoplasma detection kit (Lonza, 675 LT07-418).

676 Plasmid constructions and lentiviral particle Production: cDNA for human GS was 677 obtained from Origene. Silent mutations were introduced to make the GS cDNA resistant 678 to the GS-specific shRNA (see below, TRCN0000045628). Point-mutated constructs were 679 generated with Stratagene's QuickChange site-directed mutagenesis kit following 680 manufacturer's guidelines. The cDNA for RHOJ-EGFP (GFP-TCL) was a gift from 681 Channing Der (Addgene plasmid \# 23231) ${ }^{23}$ and was used as a template to generate the $682 \mathrm{~N}$-terminal truncated $\triangle \mathrm{N} 20-\mathrm{RHOJ}-E G F P$, lacking the first 20 amino acids and FLAG683 tagged RHOJ. Standard cloning techniques were used to fuse GS to the photoswitchable 684 fluorescent protein mEOS (pRSETa-mEos2 was a gift from Loren Looger; Addgene 685 plasmid \# 20341) ${ }^{28}$. The BiFC vector allowing simultaneous expression of two separate 686 cDNAs fused to EGFP subfragment 1 (N-terminal; containing amino acids 1 to 158) or 687 subfragment 2 (C-terminal; containing amino acids from 159 onwards) respectively was a 688 kind gift of Prof. Hideaki Mizuno (KU Leuven). GS was fused to the N-terminal 689 subfragment of EGFP and RHOJ was fused to the C-terminal EGFP subfragment to 690 generate GS-EGFP ${ }^{1 / 2}$, RHOJ-EGFP ${ }^{2 / 2}$. Lentiviral expression constructs were obtained by 691 cloning the respective cDNAs into pRRLsinPPT.CMV.MCS MM WPRE-vector. Validated 692 GS-specific (TRC clones TRCN0000045628 (used in the majority of the experiments and 693 indicated as $\mathrm{GS}^{\mathrm{KD} 1}$ in Extended Data Fig. 2a) and TRCN0000045631 (indicated as GS ${ }^{\mathrm{KD} 2}$ 694 in Extended Data Fig. 2a and only used to confirm the migration and lamellipodial defect in 695 Extended Data Fig. 3a-b) and RHOJ-specific (TRCN0000047606) shRNAs were either 
used in the pLKO.1 vector or subcloned into the pLVX-shRNA2 vector (No. PT4052-5;

697 Clontech, Westburg BV, Leusden, the Netherlands). Scrambled shRNAs or the empty vectors were used as negative controls (both with the same outcome). All constructs were sequence verified. Lentiviral particles were produced in HEK293T cells as previously described ${ }^{2}$.

701 ReCombinant ProteIn Production: Template vectors pRRLhGS, pRRLhGS ${ }^{R 324 C}$ and 702 pRRLhGS ${ }^{R 341 C}$ containing the gene encoding wild type or point mutated human GS were 703 used as templates for PCR-based cloning. Recombinant constructs were expressed in the 704 Escherichia coli strain BL21 codon + pICA2 that was transformed with pLH36-hGS in 705 which expression is induced by isopropyl b-D-1-thiogalactopyranoside under control of a 706 pL-promotor developed by the Protein Core of VIB (WO 98/48025, WO 04/074488). The 707 708 pLH36 plasmid is provided with a His 6 -tag followed by a murine caspase- 3 site. The 709 murine caspase-3 site can be used for the removal of the His 6 -tag attached at the Nterminus of the protein of interest during purification. The transformed bacteria were grown in $200 \mathrm{ml}$ Luria Bertani medium supplemented with ampicillin $\left(100 \mu \mathrm{g} \mathrm{ml}^{-1}\right)$ and kanamycin (50 $\mathrm{gg} \mathrm{ml}^{-1}$ ) overnight at $28^{\circ} \mathrm{C}$ before $1 / 100$ inoculation in a $20 \mathrm{I}$ fermenter provided with 712 Luria Bertani medium supplemented with ampicillin $\left(100 \mathrm{\mu g} \mathrm{ml}^{-1}\right)$ and $1 \%$ glycerol. The 713 initial stirring and airflow was $200 \mathrm{rpm}$ and $1.5 \mathrm{I} \mathrm{min}^{-1}$, respectively. Further, this was 714 automatically adapted to keep the $\mathrm{pO}_{2}$ at $30 \%$. The temperature was kept at $28{ }^{\circ} \mathrm{C}$. The 715 cells were grown to an optical density of $A_{600 n m}=1.0$, transferred at $20^{\circ} \mathrm{C}$, and expression was induced by addition of $1 \mathrm{mM}$ isopropyl b-D-1-thiogalactopyranoside overnight. Cells 717 were then harvested and frozen at $-20^{\circ} \mathrm{C}$. After thawing, the cells were resuspended at 3 $718 \mathrm{ml} \mathrm{g}^{-1}$ in $50 \mathrm{mM}$ Hepes $\mathrm{pH} 7.5,500 \mathrm{mM} \mathrm{NaCl}, 20 \mathrm{mM}$ imidazole, $1 \mathrm{mM}$ phenyl719 methylsulfonyl fluoride, $10 \%$ glycerol, $5 \mathrm{mM} \beta$-mercaptoethanol, $1 \mathrm{mg}$ per $100 \mathrm{ml}$ DNAsel 720 (Roche) and 1 tablet per $100 \mathrm{ml}$ Complete Protease Inhibitor (Roche). The cytoplasmic 721 fraction was prepared by using the Emulsiflex followed by centrifugation. All steps were 
conducted at $4{ }^{\circ} \mathrm{C}$. The clear supernatant was applied to a $20 \mathrm{ml} \mathrm{Ni-Sepharose} 6 \mathrm{FF}$

723 column (GE Healthcare), equilibrated with $50 \mathrm{mM}$ Hepes pH7.5, $500 \mathrm{mM} \mathrm{NaCl}, 20 \mathrm{mM}$

724 imidazole, $10 \%$ glycerol, $5 \mathrm{mM}$-mercaptoethanol and $1 \mathrm{mM}$ phenyl-methylsulfonyl

725 fluoride. The column was eluted with $50 \mathrm{mM}$ Hepes $\mathrm{pH} 7.5,500 \mathrm{mM} \mathrm{NaCl}, 400 \mathrm{mM}$

726 imidazole, $10 \%$ glycerol, $5 \mathrm{mM} \beta$-mercaptoethanol and $1 \mathrm{mM}$ phenyl-methylsulfonyl

727 fluoride after an intermediate elution step with $50 \mathrm{mM}$ imidazole in the same buffer. Finally,

728 the elution fraction was injected on a HiLoad 26/60 Superdex prep grade with $20 \mathrm{mM}$

729 Hepes $\mathrm{pH}$ 7.5, $300 \mathrm{mM} \mathrm{NaCL}, 10 \%$ glycerol and $0.5 \mathrm{mM}$ TCEP as running solution. The

730 obtained elution fractions were analyzed by SDS-PAGE. Recombinant protein

731 concentration was determined using the Micro-BCA assay (Pierce).

732 IN VITRO KNOCK-DOWN/OVEREXPRESSION STRATEGIES: To minimize off-target effects and 733 other silencing artifacts, key findings were confirmed with at least two independent and 734 validated GS-specific shRNAs (see above) and appropriate controls or with a GS-specific 735 siRNA duplex (5'-GGAAUAGCAUGUCACUAAAGCAGGC-3') and scrambled control 736 (TriFECTa ${ }^{\mathrm{TM}}$, IDT). For lentiviral transduction of shRNAs or overexpressing constructs an $737 \mathrm{MOI}$ of 10 or 5 was used, respectively. In case of simultaneous transduction of 2 different 738 shRNAs, a MOI 7.5 was used for each individual shRNA. In case of simultaneous 739 transduction of a shRNA in combination with an overexpression construct, the shRNA was 740 transduced at $\mathrm{MOI} 10$ and the overexpression construct at $\mathrm{MOI} 5$, except for 741 overexpression constructs for shRNA-resistant GS which were transduced at MOI 2.5. 742 Transductions were performed on day 0 in the evening, cells were refed with fresh medium 743 on day 1 in the morning and experiments were performed from day 3 or 4 onwards. siRNA 744 transfection mixtures (in a total volume of $500 \mu \mathrm{l}$ ) were prepared in Opti-MEM containing 745 GlutaMAX-I (Invitrogen) with Lipofectamine RNAi Max transfection reagent (Invitrogen, 746 Belgium) according to the manufacturer's instructions. The mixtures were added to the 747 cells $(150,000$ cells in 6 well-format plate) together with 2 ml EBM2 without antibiotics for 
overnight transfection after which the medium was changed back to the regular M199

749 culture medium. siRNA transfection was done at least $48 \mathrm{~h}$ prior to functional assays. BiFC

750 plasmids were transfected into HEK293T cells with Fugene ${ }$ HD transfection reagent

751 following the manufacturer's guidelines. KD efficiency and overexpression levels were

752 closely monitored for each experiment either on mRNA (QRT-PCR) or protein level.

753 RNA ISOLATION AND GENE EXPRESSION ANALYSIS: Total RNA was extracted with Invitrogen'S

754 PureLink RNA mini kit according to the manufacturer's instructions; quality and quantity 755 were measured on a Nanodrop (Thermo Scientific). cDNA synthesis was performed with 756 the iScript cDNA synthesis kit (BioRad). Quantitative RT-PCR analyses were performed as 757 previously described ${ }^{1}$ on an Applied Biosystems 7500 Fast device with in house-designed 758 primers and probes or premade primer sets (Applied Biosystems or Integrated DNA 759 Technologies) for which sequences and/or primer set ID numbers are available upon 760 request. ENOX2 or HPRT were used as housekeeping genes.

761 Western blotting AND (Co-)IMmunOpRecipitation: Proteins were extracted in Laemmli 762 buffer (125 mM Tris-HCl (pH 6.8), 2\% SDS,10\% glycerol) or in RIPA buffer (25 mM Tris$763 \mathrm{HCl}(\mathrm{pH} 7.6), 150 \mathrm{mM} \mathrm{NaCl}, 1 \% \mathrm{NP}-40,1 \%$ sodium deoxycholate, $0.1 \%$ SDS) containing 764 protease and phosphatase inhibitor mixes (Roche Applied Science). After shearing of 765 genomic DNA, proteins in the lysates were separated by SDS-PAGE, transferred to 766 nitrocellulose or polyvinylidene difluoride membranes and detected with specific antibodies 767 and HRP-conjugated secondary antibodies in combination with ECL or SuperSignal Femto 768 Western blotting substrate (Thermo Scientific). Signal was acquired with Image Quant LAS $7694000 \vee 1.2$ and densitometric quantification was done with ImageJ. For MLC and pMLC 770 immunoblotting, each sample was loaded on two separate gels. One gel was used to 771 detect MLC and the second was used to detect pMLC. Both gels had their own loading 772 control, namely $\alpha$-tubulin. pMLC/MLC was quantified as follows: (pMLC/ $\alpha$-tubulin)/(MLC/ $\alpha-$ 
773 tubulin), abbreviated in the figure panel as (c)pMLC/(c)MLC with (c) meaning "corrected for

774 corresponding loading control'. Membrane versus cytosolic protein fractions were purified 775 with the Plasma Membrane Protein Extraction Kit (101Bio) according to the manufacturer's 776 guidelines and using proprietary buffers. For co-immunoprecipitation (co-IP) of 777 endogenous or overexpressed proteins, ECs were lysed by rotating at $4{ }^{\circ} \mathrm{C}$ during at least $7784 \mathrm{~h}$ in co-IP lysis buffer $(20 \mathrm{mM}$ Tris-HCl pH8, $137 \mathrm{mM} \mathrm{NaCl}, 10 \%$ glycerol, $1 \%$ nonidet 779 NP-40 and 2 mM EDTA). Equal amounts of protein were incubated overnight with specific 780 antibodies or matching isotype control IgGs at $4{ }^{\circ} \mathrm{C}$. Subsequently, $20 \mu$ of protein A/G781 Sepharose beads was added to the immune complexes for $4 \mathrm{~h}$ at $4{ }^{\circ} \mathrm{C}$ under gentle 782 rotation. The beads were pelleted, washed three times with ice-cold co-IP lysis buffer and 783 boiled for $5 \mathrm{~min}$ in reducing agent and loading buffer prior to SDS-PAGE. To determine the 784 impact of deleting RHOJ's first $20 \mathrm{~N}$-terminal AAs on the interaction with GS, co-IPs were 785 done as above on ECs simultaneously overexpressing GS and RHOJ-EGFP or $\Delta$ N20786 RHOJ-EGFP. In some of the experiments the expression of the $\triangle$ N20-RHOJ-EGFP was 787 lower than the expression of RHOJ-EGFP. To correct for this possible bias, densitometric 788 quantification of all bands was performed in ImageJ and signals in the IP lanes were 789 normalized to the input signals. The amount of GS IP'ed was the same in the RHOJ-EGFP 790 and $\Delta$ N20-RHOJ-EGFP condition (data not shown).

791 BIOCHEMICAL AND METABOLIC ASSAYS: BICINCHONINIC ACID (BCA) ASSAY (Pierce) was used to 792 determine protein content with Gen5 1.11.5 (BioTek Instruments). LDH RELEASE as a 793 measure for cell survival was determined with the Cytotoxicity Detection Kit (Roche 794 Applied Science) with Gen5 1.11.5 (BioTek Instruments). INTRACELLULAR REACTIVE OXYGEN 795 SPECIES (ROS) LEVELS were determined by $\mathrm{CM}-\mathrm{H}_{2}$ DCFDA dye (Invitrogen) labeling 796 following manufacturer's guidelines. GLUTAMINE SYNTHETASE ACTIVITY in living cells. The 797 enzyme activity in living cells was determined by pulse-labeling the cells for 30 min with 2 $798 \mathrm{mM}^{15} \mathrm{NH}_{4} \mathrm{Cl}$ and subsequent determination of ${ }^{15} \mathrm{~N}$ incorporation in intracellular glutamine 
799 by gas chromatography - mass spectrometry GC-MS (see below). Similarly, GS activity 800 was measured by pulse-labeling for $30 \mathrm{~min}$ with $0.5 \mathrm{mM}\left[\mathrm{U}-{ }^{13} \mathrm{C}\right]$-glutamic acid and 801 subsequent tracing of ${ }^{13} \mathrm{C}$ into glutamine by GC-MS. The $0.025 \mathrm{mM}$ glutamine condition 802 was added to this assay for the sole purpose of having a positive control - lowering 803 external glutamine levels should increase GS activity - and are not in any way reflecting 804 maximal GS activity. Background signals were determined by pre-incubating the cells with 805 the GS inhibitor MSO. As an independent manner (not relying on labeling one of the 806 immediate substrates $\left(\mathrm{NH}_{4}{ }^{+}\right.$or glutamate)) to determine GS activity, we performed steady 807 state labeling of ECs with $\left[\mathrm{U}-{ }^{13} \mathrm{C}\right]$-glucose $(5.5 \mathrm{mM})$ and determined carbon contribution to $808 \alpha$-ketoglutarate, glutamate and glutamine (for labeling scheme see Extended Data Fig. 5f). 809 Prior to derivatization for GC-MS analysis, cells were washed with ice-cold $0.9 \% \mathrm{NaCl}$ and 810 extracted in ice cold 80/20 methanol/water. GLUTAMINE UPTAKE ASSAY: Dynamic [U- ${ }^{13}$ C]811 glutamine uptake assays were performed as follows: $2.5 \times 10^{5}$ cells/well were seeded in 6 812 well plates and pulse-labeled for 0, 0.5,10, 20 and $30 \mathrm{~min}$ with the regular M199 culture 813 medium containing $0.6 \mathrm{mM}\left[\mathrm{U}-{ }^{13} \mathrm{C}\right]$-glutamine instead of the regular $0.6 \mathrm{mM}$ unlabeled 814 glutamine. The 0 min time point represents an absolute negative control for which extracts 815 were made from ECs that were never treated with tracer-containing medium. For the 0.5 816 min time point, the labeled medium was put on the cells and immediately aspirated (all 817 together taking $0.5 \mathrm{~min}$ ). At all time points, cells were thoroughly washed twice with ice818 cold $0.9 \% \mathrm{NaCl}$ to ensure complete removal of tracer-containing medium. Cellular extracts 819 were then made in ice-cold 80/20 methanol/water, prior to derivatization for GC-MS 820 measurements. Alternatively, cells were incubated with $0.5 \mu \mathrm{Ci} \mathrm{ml}^{-1}\left[\mathrm{U}-{ }^{14} \mathrm{C}\right]$-L-glutamine for 82110 min after which they were washed at least three times with ice-cold PBS. The last PBS 822 wash was collected and checked for residual radioactivity. Cells were then lysed with 200 $823 \mu \mathrm{l} 0.2 \mathrm{~N} \mathrm{NaOH}$ and lysates were neutralized with $20 \mu \mathrm{l} 1 \mathrm{~N} \mathrm{HCl}$ and used for scintillation 824 counting. ${ }^{3}$ H]-THYMIDINE INCORPORATION: Proliferation was determined by labeling the cells 
825 with $1 \mu \mathrm{Ci} \mathrm{ml}{ }^{-1}\left[{ }^{3} \mathrm{H}\right.$ ]-thymidine for $2 \mathrm{~h}$, followed by fixation in $100 \%$ ethanol for $15 \mathrm{~min}$, 826 precipitation with $10 \%$ trichloroacetic acid and finally lysis in $0.1 \mathrm{~N} \mathrm{NaOH}$. Scintillation 827 counting was used to assess the amount of $\left[{ }^{3} \mathrm{H}\right]$-thymidine incorporated into the DNA. 828 ENERGY CHARGE ASSESSMENT: $1.5 \times 10^{6}$ cells were collected in $100 \mu$ ice cold $0.4 \mathrm{M}$ 829 perchloric acid containing $0.5 \mathrm{mM}$ EDTA. $\mathrm{pH}$ was adjusted with $100 \mu \mathrm{l}$ of $2 \mathrm{M} \mathrm{K}_{2} \mathrm{CO}_{3} .100$ $830 \mu \mathrm{l}$ of the mixture was subsequently injected onto an Agilent 1260 HPLC with a C18831 Symmetry column ( $150 \times 4.6 \mathrm{~mm} ; 5 \mathrm{~mm}$; Waters $)$, thermostated at $22.5^{\circ} \mathrm{C}$. Flow rate was

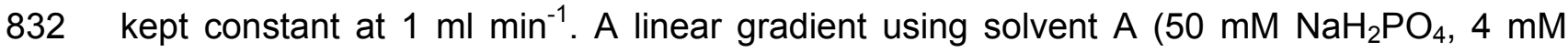
833 tetrabutylammonium, adjusted to $\mathrm{pH} 5.0$ with $\left.\mathrm{H}_{2} \mathrm{SO}_{4}\right)$ and solvent $\mathrm{B}\left(50 \mathrm{mM} \mathrm{NaH}_{2} \mathrm{PO}_{4}, 4\right.$ $834 \mathrm{mM}$ tetrabutylammonium, $30 \% \mathrm{CH}_{3} \mathrm{CN}$, adjusted to $\mathrm{pH} 5.0$ with $\mathrm{H}_{2} \mathrm{SO}_{4}$ ) was accomplished 835 as follows: $95 \%$ A for $2 \mathrm{~min}$, from 2 to $25 \mathrm{~min}$ linear increase to $100 \% \mathrm{~B}$, from 25 to $27 \mathrm{~min}$ 836 isocratic at $100 \% \mathrm{~B}$, from 27 to 29 min linear gradient to $95 \%$ A and finally from 29 to 35 $837 \mathrm{~min}$ at $95 \%$ A. ATP, ADP and AMP were detected at $259 \mathrm{~nm}$. SEAHORSE EXTRACELLULAR 838 FLUX MEASUREMENTS: ECs were seeded at $1.5 \times 10^{5}$ cells per well on Seahorse XF24 839 tissue culture plates (Seahorse Bioscience Europe). Oxygen consumption (OCR) 840 measurements were performed at 6 min intervals (2 min mixing, 2 min recovery, 2 min 841 measuring) in a Seahorse XF24 device (XF Reader 1.8.1.1 software). Consecutive 842 treatments with oligomycin $(1.2 \mu \mathrm{M}$ final), FCCP $(5 \mu \mathrm{M}$ final) and antimycin A (1 $\mu \mathrm{M}$ final) 843 were performed to allow quantification of ATP-coupled OCR (OCR $\mathrm{ATP}$ ) and maximal 844 respiration, next to basal OCR (OCR bas). GLYCOLYTIC FLUX: ECs were cultured for $6 \mathrm{~h}$ in 845 medium containing $0.4 \mathrm{mCi} \mathrm{m}^{-1}\left[5^{3} \mathrm{H}\right]-\mathrm{D}$-glucose (Perkin Elmer) after which supernatant 846 was transferred into glass vials sealed with rubber stoppers. ${ }^{3} \mathrm{H}_{2} \mathrm{O}$ was captured in 847 hanging wells containing a Whatman paper soaked with $\mathrm{H}_{2} \mathrm{O}$ over a period of $48 \mathrm{~h}$ at 37 $848{ }^{\circ} \mathrm{C}$ to reach saturation ${ }^{1}$. Then the paper was used for liquid scintillation counting 849 (QuantaSmart TM V4 PerkinElmer). ${ }^{14} \mathrm{C}$-GLUCOSE OXIDATION: ECs were incubated for $6 \mathrm{~h}$ 850 in medium containing $0.55 \mathrm{mCi} \mathrm{ml}^{-1}\left[6-{ }^{14} \mathrm{C}\right]-\mathrm{D}$-glucose. After that, $250 \mu \mathrm{l}$ of $2 \mathrm{M}$ perchloric 
851 acid was added to each well to stop cellular metabolism and to release ${ }^{14} \mathrm{CO}_{2}$, which was 852 captured overnight at room temperature in 1x hyamine hydroxide-saturated Whatman 853 paper. The radioactivity in the paper was determined by liquid scintillation counting 854 (QuantaSmart TM V4 PerkinEImer) ${ }^{1} \cdot{ }^{14}$ C-GLUTAMINE OXIDATION: ECs were incubated for 6 $855 \mathrm{~h}$ with medium containing $0.5 \mathrm{mCi} \mathrm{ml}^{-1}\left[\mathrm{U}-{ }^{14} \mathrm{C}\right]$-glutamine. $250 \mathrm{ml}$ of $2 \mathrm{M}$ perchloric acid was added to the cells to stop cellular metabolism and release ${ }^{14} \mathrm{CO}_{2}$. Trapping of ${ }^{14} \mathrm{CO}_{2}$ 857 occurred as described above for glucose oxidation ${ }^{1}$.

Protein (auto)palmitoylation detection: IN Vitro palmitoylation (CLICK REACtion$B A S E D):$ Purified bacterial GS protein was incubated with the indicated concentration of palmitoyl alkyne-coenzyme A (Cayman Chemical) for $6 \mathrm{~h}$ at room temperature. The GS protein was then denatured by the addition of SDS. A click reaction with azide-biotin was performed to label the palmitoylated proteins ${ }^{25}$. Palmitoylated proteins were detected by 863 SDS-PAGE followed by blotting with streptavidin-horseradish peroxidase. FLUORESCENCEBASED COA RELEASE DETECTION: During autopalmitoylation of proteins, palmitate is transferred from palmitoyl-CoA to the protein thereby releasing reduced CoA. $\alpha-$ Ketoglutarate dehydrogenase can use CoA to convert a-ketoglutarate to succinyl-CoA, a 867 reaction that features reduction of $\mathrm{NAD}^{+}$to fluorescent $\mathrm{NADH}^{29}$. In brief, recombinant 868 human GS was incubated with palmitoyl-CoA in MES buffer at physiological pH for at least $8691 \mathrm{~h}$ at $30{ }^{\circ} \mathrm{C}$. The volume was then adjusted to $200 \mu \mathrm{l}$ in $50 \mathrm{mM}$ sodium phosphate buffer

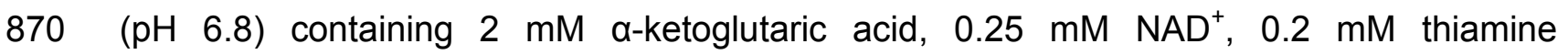
871 pyrophosphate, $1 \mathrm{mM}$ EDTA, $1 \mathrm{mM}$ DTT and $32 \mathrm{mU}$ a-ketoglutarate dehydrogenase. $872 \mathrm{NADH}$ levels were measured at $20 \mathrm{~min}$ after initiation of the reaction on a VICTOR plate 873 reader (340 $\mathrm{nm}$ excitation - $465 \mathrm{~nm}$ emission). The experiment was performed in two 874 directions: either with varying doses of palmitoyl-CoA for a fixed amount of recombinant 875 GS or with varying amounts of recombinant GS for a fixed concentration of palmitoyl-CoA $876(40 \mu \mathrm{M})$. AFFINITY CHROMATOGRAPHY: A previously published protocol was used to 
877 determine cell-free binding of recombinant human GS to palmitoyl-CoA agarose ${ }^{30}$. A total 878 of $50 \mu \mathrm{l}$ of immobilized palmitoyl-CoA-agarose was equilibrated with $20 \mathrm{mM}$ Tris- $\mathrm{HCl}(\mathrm{pH}$ $8798.4 / 120 \mathrm{mM} \mathrm{NaCl}$. The beads were incubated with $40 \mu \mathrm{g}$ of recombinant human $\mathrm{GS}$ in a 880 final volume of $200 \mu \mathrm{l}$ for $2 \mathrm{~h}$ at room temperature on a rotatory system. Beads were 881 pelleted and $20 \mu \mathrm{l}$ of the supernatant was collected as the flow through (FT) fraction. 882 Beads were then washed eight times with $500 \mu \mathrm{l}$ of $20 \mathrm{mM}$ Tris- $\mathrm{HCl}(\mathrm{pH} 8.4) / 120 \mathrm{mM} \mathrm{NaCl}$ 883 buffer. $20 \mu \mathrm{l}$ of the last wash fraction was collected as fraction W8. Beads were then eluted 884 with SDS loading buffer and heated for $15 \mathrm{~min}$ at $60^{\circ} \mathrm{C} .2 \mu \mathrm{g}$ of recombinant protein was 885 used as input fraction (IF). IF, FT, W8 and SDS-eluate were analysed by immunoblotting 886 for GS. IN CELL LABELING: In cell labeling experiments were performed essentially as 887 described previously ${ }^{25}$. HEK-293T cells were transfected with the indicated expression 888 plasmids. Twenty-four $\mathrm{h}$ after transfection, the medium was replaced with DMEM $+10 \%$ 889 dialyzed FBS containing the indicated probes (50 $\mu \mathrm{M} 16 \mathrm{C}-\mathrm{YA}$ or $50 \mu \mathrm{M}$ 16C-BYA). After $89018 \mathrm{~h}$, cell lysates were collected by incubation of the cells on ice for $15 \mathrm{~min}$ in lysis buffer 891 (50 mM TEA-HCl (pH=7.4), 150 mM NaCl, 1\% Triton X-100, 0.5\% sodium deoxycholate, $8920.1 \%$ SDS and $5 \mathrm{mM}$ PMSF) followed by centrifugation for $10 \mathrm{~min}$ at $15,000 \mathrm{~g}$. Equal 893 amounts of protein were then used for a click reaction with azide-biotin. For labeling with 894 17-ODYA, FLAG-RHOJ overexpressing ECs were incubated overnight with 17-ODYA (50 $895 \mu \mathrm{M})$ in M199 supplemented with 3.6\% fatty acid free BSA, 10\% dialyzed FBS and $5 \mathrm{mM}$ 896 sodium pyruvate. Cells were washed with ice-cold PBS and lysed in NaP lysis buffer (0.2 $\left.897 \mathrm{M} \mathrm{Na}_{2} \mathrm{HPO}_{4} .2 \mathrm{H}_{2} \mathrm{O}, 0.2 \mathrm{M} \mathrm{NaH}_{2} \mathrm{PO}_{4} .2 \mathrm{H}_{2} \mathrm{O}, 1 \mathrm{M} \mathrm{NaCl}, 10 \% \mathrm{NP} 40\right)$. $2 \mu \mathrm{g}$ of anti-Flag 898 antibody was conjugated to $20 \mu$ l of dynabeads protein $\mathrm{G}$ (Thermofisher) for $1 \mathrm{~h}$ at RT. 899 After washing the beads twice with NaP lysis buffer, at least $500 \mu \mathrm{g}$ of protein was added 900 to the beads for $3 \mathrm{~h}$ at $4{ }^{\circ} \mathrm{C}$. Then beads were washed 3 times with NaP lysis buffer and 901 resuspended in $20 \mu \mathrm{l}$ of resuspension buffer (4\% SDS, $50 \mathrm{mM}$ TEA, $150 \mathrm{mM} \mathrm{NaCl}$ ). The 902 click reaction was initiated by adding $0.5 \mu \mathrm{l}$ of $5 \mathrm{mM}$ tetramethylrhodamine azide (TAMRA) 
903 (Lumiprobe), $0.5 \mu \mathrm{l} 50 \mathrm{mM}$ tris(2-carboxyethyl)phosphine hydrochloride (TCEP-HCl), $0.5 \mu \mathrm{l}$

$90410 \mathrm{mM}$ tris[(1-benzyl-1H-1,2,3-triazol-4-yl)methyl]amine (TBTA) and $2.4 \mu \mathrm{l}$ of $5 \mathrm{mM}$ freshly 905 made ascorbic acid. Samples were then incubated for $1 \mathrm{~h}$ at $37^{\circ} \mathrm{C}$ in the dark. Sample 906 buffer $(9.4 \mu \mathrm{l})$ and reducing agent $(3.7 \mu \mathrm{l})$ were added to stop the reaction. After $10 \mathrm{~min}$ at 907 room temperature in the dark, samples were frozen at $-80{ }^{\circ} \mathrm{C}$ or run on a $10 \%$ Bis-TRIS 908 gel in MES buffer. In-gel fluorescence was imaged with Typhoon TM FLA 9500 V1.0. 909 STREPTAVIDIN-PULLDOWN: After click reaction with azide-biotin, free azide-biotin was 910 removed from the samples by centrifugal filtration column (Millipore). The samples were 911 then incubated with streptavidin-conjugated beads for $1 \mathrm{~h}$ at room temperature. After 912 washing with PBS-T, proteins were eluted from the beads by incubation in elution buffer $913(95 \%$ formamide, $10 \mathrm{mM}$ EDTA $(\mathrm{pH}=8.0))$ at $95{ }^{\circ} \mathrm{C}$ for $5 \mathrm{~min}$. ACYL-RESIN-ASSISTED 914 CAPTURE (ACYL-RAC) in which free cysteine thiols are chemically blocked and palmitoylated 915 cysteines are exposed and captured by a resin, was performed with the CAPTUREome ${ }^{T M}$ 916 S-Palmitoylated Protein Kit (Badrilla) with minor adaptations to the manufacturer's 917 guidelines. $500 \mu \mathrm{g}$ of protein were incubated for $4 \mathrm{~h}$ in $500 \mu \mathrm{l}$ of thiol blocking reagent (to 918 block free thiols). Proteins were precipitated with ice-cold acetone and afterwards 919 solubilized with $300 \mu \mathrm{l}$ of binding buffer and spun down. After protein quantification, $30 \mu \mathrm{g}$ 920 was kept as total input fraction (IF), and equal amounts of protein were incubated for $2.5 \mathrm{~h}$ 921 with (or without to obtain the negative control preserved bound fraction (pBF)) a thioester 922 linkage specific cleavage reagent to cleave the thioester bond. Newly liberated thiols were 923 captured with CAPTUREome ${ }^{\mathrm{TM}}$ resin. The resin was spun down and the supernate was 924 collected as the cleaved unbound fraction (cUF) to check if the proteins of interest were 925 indeed completely depleted from the thioester cleavage reagent (meaning efficient capture 926 of the free thiols by the resin). After thorough washing of the resin, captured proteins 927 (cleaved bound fraction (cBF)), were eluted with reductant and analyzed together with the 928 IF, cUF and pBF by SDS-PAGE followed by immunoblotting. 
930 GC-MS ANALYSIS: Metabolites from cells were extracted in $800 \mu \mathrm{l} 80 \%$ methanol (at -80 $\left.931{ }^{\circ} \mathrm{C}\right)$. Next the extracts were centrifuged at $4{ }^{\circ} \mathrm{C}$ for $15 \mathrm{~min}$ at $20,000 \times \mathrm{g}$ and the 932 supernatants were dried in a vacuum centrifuge. $25 \mu$ of a $2 \%$ methoxyamine 933 hydrochloride solution (20 mg dissolved in $1 \mathrm{ml}$ pyridine) was added to the dried fractions 934 which were then incubated at $37^{\circ} \mathrm{C}$ for $90 \mathrm{~min}$. Then $75 \mu \mathrm{l}$ of $\mathrm{N}$-tert-butyldimethylsilyl-N935 methyltrifluoroacetamide with $1 \% \mathrm{~N}$-tert-butyldimethyl-chlorosilane (Sigma-Aldrich) was 936 added and the reaction was carried out for $30 \mathrm{~min}$ at $60{ }^{\circ} \mathrm{C}$. Reaction mixtures were 937 centrifuged for $15 \mathrm{~min}$ at $20,000 \times \mathrm{g}$ at $4{ }^{\circ} \mathrm{C}$ in order to remove insolubilities and the 938 supernatant was transferred to a glass vial with conical insert (Agilent). GC-MS analyses 939 were performed on an Agilent 7890A GC equipped with a HP-5 ms 5\% Phenyl Methyl 940 Silox (30 m - 0.25 mm i.d. - $0.25 \mu \mathrm{m}$; Agilent Technologies) capillary column, interfaced 941 with a triple quadrupole tandem mass spectrometer (Agilent 7000B, Agilent Technologies) 942 operating under ionization by electron impact at $70 \mathrm{eV}$. The injection port, interface and ion 943 source temperatures were kept at $230{ }^{\circ} \mathrm{C}$. Temperature of the quadrupoles was kept at $944150{ }^{\circ} \mathrm{C}$. The injection volume was $1 \mu \mathrm{l}$, and samples were injected at 1:10 split ratio. 945 Helium flow was kept constant at $1 \mathrm{ml} \mathrm{min}^{-1}$. The temperature of the column started at 100 $946{ }^{\circ} \mathrm{C}$ for $5 \mathrm{~min}$ and increased to $260{ }^{\circ} \mathrm{C}$ at $2{ }^{\circ} \mathrm{C} \mathrm{min}{ }^{-1}$. Next, a $40{ }^{\circ} \mathrm{C} \mathrm{min}{ }^{-1}$ gradient was 947 carried out until the temperature reached $300{ }^{\circ} \mathrm{C}$. After the gradient, the column was 948 heated for another $3 \mathrm{~min}$ at $325^{\circ} \mathrm{C}$. The GC-MS analyses were performed in Single lon 949 Monitoring (SIM) scanning for the isotopic pattern of metabolites.

950 LC-MS ANALYSIS: POLAR METABOLITES were extracted using $250 \mu \mathrm{l}$ of a 50-30-20 951 (methanol-acetonitrile-10 mM ammonium acetate $\mathrm{pH} 9.3$ containing $2 \mu \mathrm{M}$ of deuterated 952 (d27) myristic acid as internal standard) extraction buffer. Following extraction, precipitated 953 proteins and insolubilities were removed by centrifugation at $20,000 \times \mathrm{g}$ for $20 \mathrm{~min}$ at $4{ }^{\circ} \mathrm{C}$. 954 The supernatant was transferred to the appropriate mass spectrometer vials. 
955 Measurements were performed using a Dionex UltiMate 3000 LC System (Thermo

956 Scientific) in-line connected to a Q-Exactive Orbitrap mass spectrometer (Thermo 957 Scientific). $15 \mu \mathrm{l}$ of sample was injected and loaded onto a Hilicon iHILIC-Fusion(P) 958 column (Achrom). A linear gradient was carried out starting with $90 \%$ solvent A (LC-MS 959 grade acetonitrile) and 10\% solvent B (10 mM ammonium acetate pH 9.3). From 2 to 20 960 mins the gradient changed to $80 \%$ B and was kept at $80 \%$ until $23 \mathrm{~min}$. Next a decrease to $96140 \%$ B was carried out to $25 \mathrm{~min}$, further decreasing to $10 \%$ B at $27 \mathrm{~min}$. Finally, 10\% B 962 was maintained until $35 \mathrm{~min}$. The solvent was used at a flow rate of $200 \mu \mathrm{min}^{-1}$, the 963 column's temperature was kept constant at $25^{\circ} \mathrm{C}$. The mass spectrometer operated in 964 negative ion mode, settings of the HESI probe were as follows: sheath gas flow rate at 35 , 965 auxiliary gas flow rate at 10 (at a temperature of $260^{\circ} \mathrm{C}$ ). Spray voltage was set at $4.8 \mathrm{kV}$, 966 temperature of the capillary at $300{ }^{\circ} \mathrm{C}$ and S-lens RF level at 50. A full scan (resolution of 967140,000 and scan range of $\mathrm{m} / \mathrm{z} 70-1050$ ) was applied. For the data analysis, we used an 968 in-house library and metabolites of interest were quantified (area under the curve) using 969 the XCalibur 4.0 (Thermo Scientific) software platform.

970 IN VITRO ASSAYS: ENDOTHELIAL SPHEROID CAPILLARY SPROUTING was performed following 971 established protocols ${ }^{1,2}$. To form the spheroids, ECs were cultured overnight in hanging 972 drops in EGM2 medium with methylcellulose (Sigma-Aldrich; 20 \%volume of a 1.2\% 973 solution of methylcellulose $4000 \mathrm{cP}$ ). Spheroid sprouting entails both EC proliferation and 974 migration. To have a 'clean' view on the migration aspect in sprouting, we also included 975 conditions in which we blocked EC proliferation prior to sprout formation. More in

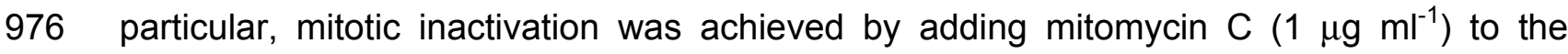
977 medium. To induce sprouting, spheroids were embedded in a collagen gel and incubated 978 for $20 \mathrm{~h}$. If required, chemical compounds (Fasudil at $10 \mu \mathrm{M}, \mathrm{H} 1152$ at $1 \mu \mathrm{M}$ and Y26732 979 at $10 \mu \mathrm{M})$ were added during the collagen gel incubation step. Spheroids were then fixed 980 with $4 \%$ paraformaldehyde and imaged under phase contrast illumination with a Motic AE 
98131 microscope (Motic Electric Group Co Ltd) or a Leica DMI6000B microscope (Leica

982 Microsystems). Phase contrast images were used to quantify the number of sprouts per 983 spheroid and the total sprout length (cumulative length of all sprouts on a spheroid). 984 Spheroid body circumference was measured to correct for differences in size of the 985 spheroid. Per experiment (ie per individual HUVEC isolation) at least 10 spheroids per 986 condition were analyzed. SCRATCH WOUND ASSAYS: 75,000 HUVECs were seeded in 24987 well format and were allowed to reach confluency over the next $24 \mathrm{~h}$. At time T0 the 988 confluent monolayer was scratched with a $200 \mu$ pipet tip and photographed. The cells 989 were further incubated for the indicated times and photographed again at time point Tx.

990 Gap area at T0 minus gap area at Tx was measured with ImageJ and expressed as \% 991 migration distance. Per well, three non-overlapping regions along the scratch were 992 analyzed. Much like the spheroid sprouting, scratch wound healing is a combined readout 993 for EC migration and proliferation. Therefore, we also included conditions in which the ECs 994 were pre-treated with mitomycin $\mathrm{C}\left(1 \mu \mathrm{g} \mathrm{ml}^{-1}\right)$ to rule out the effect of proliferation. BOYDEN 995 CHAMBER ASSAYS: 50,000 HUVECs were seeded on 0.1\% gelatin-coated transwells and 996 allowed to adhere. Then, the transwells were washed and refed with medium containing 997 only $0.1 \%$ FBS and placed in bottom wells containing medium with $5 \%$ FBS as a pro998 migratory stimulus. $16 \mathrm{~h}$ later, transwells were processed and analysed for numbers of 999 migrated cells. Pre-treatment with mitomycin C (see above) was applied. VELOCITY OF 1000 RANDOM MOVEMENT was assessed on HUVECs that were sparsely seeded on glass bottom 1001 24-well plates. Time-lapse videos were generated by confocal image acquisition at $4 \mathrm{~min}$ 1002 intervals. Velocity of movement was determined by tracking nucleus position in function of 1003 time $\left(\mu \mathrm{m} \mathrm{h}^{-1}\right)$ (Tracking Tool TM, Gradientech AB, Uppsala, Sweden). Per condition, on 1004 average 2 or 3 individual cells were traced in each biological repeat. LAMELLIPODIAL AREA 1005 was measured on sparsely seeded phalloidin-stained ECs with Leica MM AF 1006 morphometric analysis software (Leica Microsystems, Mannheim, Germany) with in-house 
1007 developed journals and is expressed in percent of total cell area. Treatment with MSO (1 $1008 \mathrm{mM}), \mathrm{Y} 27632(10 \mu \mathrm{M})$, Fasudil $(10 \mu \mathrm{M}), \mathrm{H} 1152(1 \mu \mathrm{M}), \mathrm{ML}$ (15 $\mu \mathrm{M})$ and peptide $18(15$ $1009 \mu \mathrm{M})$ were done $24 \mathrm{~h}$ prior to analysis of the cells. Per experimental condition, a minimum 1010 of ten individual cells was analyzed. STAINING AND QUANTIFICATION OF VE-CADHERIN 1011 JUNCTIONS: VE-cadherin staining and quantification of junctional length and gap index was 1012 performed as previously described ${ }^{31}$. First, the total junctional length (100\%) was 1013 determined by summing up all segments, then the sum of all continuous segments was 1014 calculated as the percentage of total junctional length. The percentage difference between 1015 total and continuous represents the discontinuous length. Gap size index (intercellular gap 1016 area/cell number) was determined with the formula ([intercellular gap area/total cell area] $1017 \times 1,000) / c e l l$ number. Junctional lengths, intercellular gap area, and total cell area were 1018 defined manually with ImageJ. For each condition, a minimum of 10 fields was quantified 1019 (10-15 cells per field on average) per experiment, and data shown represent the mean of 1020 at least 3 independent experiments. TRANS ENDOTHELIAL ELECTRICAL RESISTANCE (TEER): 102150,000 ECs were seeded on $6.5 \mathrm{~mm} 0.1 \%$ gelatin-coated polyester transwells, $0.4 \mu \mathrm{m}$ 1022 pore size (Costar ref. 3470, Sigma-Aldrich). The electrical resistance was measured with 1023 an Endhome-6 electrode (World Precisions Instruments) connected to an EVOM2 1024 voltohmmeter (World Precisions Instruments). Gelatin-coated wells without cells were 1025 used to measure the intrinsic electrical resistance of the inserts for background 1026 subtraction. Measurements were performed every day for 4 consecutive days, with at least 10272 measurements per condition.

1028 ACTIN DYNAMICS AND Rho (KINASE) ACTIVITY ASSAYS: LATRUNCULIN WASH-OUT: ECs were 1029 treated with latrunculin $B\left(100 \mathrm{ng} \mathrm{m}^{-1}\right)$ for $30 \mathrm{~min}$ and were then washed three times with 1030 culture medium. The cells were fixed at the indicated time points and stained with 1031 phalloidin to visualize actin stress fibers. THE F-/G-ACTIN RATIO in GS $^{\mathrm{KD}}$ vs control ECs was 1032 determined in $4 \%$ paraformaldehyde-fixed cells which were permeabilized for $10 \mathrm{~min}$ in 
1033 PBS with $0.2 \%$ Triton X-100 and stained with phalloidin-Alexa 488 and deoxyribonuclease 1034 I-Alexa $594(1: 200)^{32}$. Fluorescence intensities were quantified with ImageJ and were 1035 based on gray values. On average, ten individual cells were analyzed per experimental 1036 condition. For RHOJ ACTIVITY measurements, cells were lysed in buffer containing $50 \mathrm{mM}$ 1037 Tris, $\mathrm{pH} 7.6,150 \mathrm{mM} \mathrm{NaCl}, 1 \%$ Triton $\mathrm{X}-100,0.5 \mathrm{mM} \mathrm{MgCl}_{2}$, protease inhibitors and 0.1 $1038 \mu \mathrm{g} \mathrm{I}^{-1}$ biotinylated CRIB-peptide. After spinning down for $4 \mathrm{~min}$ at $14,000 \mathrm{rpm}$ at $4{ }^{\circ} \mathrm{C}, 50$ $1039 \mu \mathrm{l}$ streptavidin-coated beads were added to the lysates. Subsequently, samples were 1040 rotated for $30 \mathrm{~min}$ at $4{ }^{\circ} \mathrm{C}$, beads were washed 4 times in the above buffer after which they 1041 were boiled for $5 \mathrm{~min}$ in reducing agent and loading buffer ${ }^{33}$. As negative controls in this 1042 assay, we used lysates from RHOJ ${ }^{\mathrm{KD}} \mathrm{ECs}$, a streptavidin beads only-condition and lysates 1043 in which the biotinylated CRIB-peptide was replaced by an irrelevant biotinylated protein 1044 (Fig. 4c). RHOA/B/C ACTIVITY was determined with GST-Rhotekin pull down assays 1045 following previously established protocols ${ }^{34}$. ROCK ACTIVITY was assayed by determining 1046 phosphorylation of the ROCK target myosin light chain 2 (MLC2) on Western Blot or by 1047 immunostaining. Fluorescence intensities from immunostainings were quantified with 1048 ImageJ and were based on gray values.

1049 CONFOCAL AND HIGH RESOLUTION IMAGING: CONFOCAL IMAGING was performed on a Zeiss 1050 LSM 510 Meta NLO or Zeiss LSM 780 confocal microscope (oil objectives: x 40 with NA 1051 1.3, x 63 with NA 1.4, x 100 with NA 1.3) with ZEN 2011 software (Carl Zeiss, Munich, 1052 Germany). Within individual experiments, all images across different experimental 1053 conditions were acquired with the same settings. DORA RHOA BIOSENSOR FRET IMAGING: 1054 RHOA activity was measured in living HUVECs by monitoring yellow fluorescent protein 1055 (YFP) FRET over donor cyan fluorescent protein (CFP) intensities as described previously $1056{ }^{35}$. In brief, a Zeiss Observer Z1 microscope, with a Chroma 510 DCSP dichroic splitter, 1057 two Hamamatsu ORCA-R2 digital CCD cameras and an attached dual camera adaptor 1058 (Zeiss) controlling a 510 DCSP dichroic mirror, was used for simultaneous monitoring of 
1059 CFP and YFP emissions using filter sets ET 480/40 and ET 540/40m (Chroma 1060 Technology, Rockingham, USA), respectively. To excite the CFP donor, ET 436/20x and 1061455 DCLP dichroic mirror was used (Chroma). For FRET/CFP ratiometric processing, CFP 1062 and YFP images were processed using the MBF ImageJ collection. The images were 1063 background-subtracted, aligned and a threshold was applied. Finally, the FRET/CFP ratio 1064 was calculated and a custom lookup table was applied to generate a color-coded image, in 1065 which white and red colors illustrate high and blue colors illustrate low RHOA activities. 1066 BIFC IMAGING AND QUANTIFICATION: BiFC was evaluated using a laser scanning microscope 1067 (Fluoview FV1000, Olympus, Tokyo, Japan) equipped with a UPLSAPO 60x Oil objective 1068 (NA1.35). Before imaging cells were fixed with $4 \%(\mathrm{v} / \mathrm{v})$ paraformaldehyde and stained 1069 with DAPI (1:1,000 dilution, Invitrogen). A 488-nm laser was used for exciting EGFP while 1070 DAPI was excited using a 405-nm laser. A DM405/488/559/635 polychroic mirror was 1071 used to guide the excitation lasers to the sample. Fluorescence images of fixed cells were 1072 acquired using a sampling speed of $4 \mu$ pixel $^{-1}$. Emission light was collected at 430-470 1073 and 500-550 nm, for DAPI and EGFP, respectively. The images were acquired with a pixel 1074 size of $207 \mathrm{~nm}(1,024$ x 1,024 pixels). BiFC was first established in HEK cells expressing 1075 GS-EGFP $^{1 / 2}$ and RHOJ-EGFP ${ }^{2 / 2}$ from one expression vector, with a construct 1076 overexpressing an unfused N-terminal EGFP half-site together with RHOJ coupled to the 1077 C-terminal EGFP half-site as a negative control (data not shown). To determine the effect 1078 of deleting the first 20 amino acids in RHOJ on BiFC in ECs, separate expression 1079 constructs for GS-EGFP ${ }^{1 / 2}$, RHOJ-EGFP ${ }^{2 / 2}$ and $\Delta$ N-RHOJ-EGFP ${ }^{2 / 2}$ were used (Ext. Data 1080 Fig. 7e). Quantification of expression efficiency was done using a home-built routine in 1081 Matlab®. TIRF MICROSCOPY: A home build setup based on an inverted microscope (IX83, 1082 Olympus) was used to detect single molecules under total internal reflection (TIRF) mode. 1083 The setup was equipped with an Electron Multiplying-CCD cameras (ImagEM C9100-13; 1084 Hamamatsu Photonics, Hamamatsu, Japan) and an APON 60XOTIRF objective lens (NA 
1.49, Olympus). The GS-mEos3.2 molecules were excited with a 561-nm line from a

1086 DPSS laser (200 mW; Coherent Inc., Santa Clara, California) and converted with a 405$1087 \mathrm{~nm}$ line from a diode laser (Cube, $100 \mathrm{~mW}$; Coherent Inc., Santa Clara, California). Before

1088

1089

1090

1091

1092

1093

1094

1095

1096

1097

1098

1099

1100

1101

1102

1103

1104

1105

1106

1107

1108

1109

1110 being expanded, the laser lines were combined using a $405 \mathrm{bcm}$ dichroic mirror. The laser lines were guided onto the sample by a dichroic mirror, z488/561/633rpc. The fluorescence of the red of mEos3.2 form was detected through a long pass filter 572 (HQ572LP), in combination with a band pass filter HQ590M40-2P. All the filters were purchased from Chroma Inc. Time-lapse fluorescence images were recorded with continuous illumination at a $62.5 \mathrm{~Hz}$ acquisition rate (16 ms per frame). SINGLE PARTICLE TRACKING (SPT): For calculation of single molecule coordinates the program 'Localizer' running from Matlab was used ${ }^{36}$. After localization, the positions of a molecule detected in consecutive frames are connected to reconstruct a trajectory using home-developed software in Matlab. Coordinates presented in consecutive frames are linked to form a single trajectory when they uniquely appear in a distance smaller than $856 \mathrm{~nm}$ (corresponding to 8 pixels). Trajectories with at least 3 steps were analyzed using variational Bayes single particle tracking analysis (vbSPT), a software package for analysis of single particle diffusion trajectories, where the diffusion constants switch randomly according to a Markov process ${ }^{37}$.

MICE: GS ${ }^{E C K O}$ MICE: To obtain inducible EC-specific GS knock-out mice, GS $^{\text {lox/lox }}$ mice ${ }^{38}$ were intercrossed with VECadherin-Cre ${ }^{E R T 2} 39$ or with Pdgfb-Cre $e^{E R T 2} 40$ mice and named $\mathrm{GS}^{\mathrm{VECKO}}$ and $\mathrm{GS}^{\mathrm{pECKO}}$ respectively. Correct Cre-mediated excision of the loxed GS segment in tamoxifen-treated GS ${ }^{\mathrm{ECKO}}$ mice was confirmed via PCR analysis of genomic DNA (Extended Data Fig. 1d-e). GENERATION OF GS ${ }^{+/ G F P}$ CHIMERAS : Blastocysts were collected from superovulated C57BL/6 females at post-coital day 3.5 and were cultured for 5-8 days in ES cell culture medium consisting of Knockout DMEM medium (Invitrogen), with 2 mM L-glutamine, fetal bovine serum (Hyclone, ThermoScientific), MEM non- 
1111

1112

1113

1114

1115

1116

1117

1118

1119

1120

1121

1122

1123

1124

1125

1126

1127

1128

1129

1130

1131

1132

1133

1134

1135

1136

essential amino acids 100X (Invitrogen), $0.01 \mathrm{mM} \beta$-mercaptoethanol (Sigma-Aldrich), 1 $\mathrm{mM}$ sodium pyruvate (Invitrogen), $100 \mathrm{U} \mathrm{ml}^{-1}$ penicillin, $100 \mu \mathrm{g} \mathrm{ml}^{-1}$ streptomycin, and 2,000 $\mathrm{U} \mathrm{ml}^{-1}$ Leukemia Inhibitory Factor (Merck, Millipore). Afterwards, the inner cell mass was selectively removed from the trophectoderm, trypsinized and replated on a Mitomycin C-arrested MEF feeder monolayer. ES cells were fed every day and passaged every 2-4 days onto new feeder cells. GS ${ }^{+/ G F P}$ ES cells (E14IB10 ES cell line) ${ }^{6}$ were injected into C57BL/6 blastocysts and high chimeric pups were killed at P5 for detection of GFP in the retinal microvasculature.

IN VIVO MODELS: ANALYSIS OF DORSAL DERMAL BLOOD VESSEL NETWORK: From E11.5 to E13.5 after vaginal plug, GS ${ }^{\mathrm{vECKO}}$ pregnant dams were treated with tamoxifen $\left(50 \mathrm{mg} \mathrm{kg}{ }^{-1}\right)$ by oral gavage. At E16.5 they were euthanized by cervical dislocation after which embryos were dissected from the uterus. Yolk sacs were collected, washed with PBS and used for genotyping of the embryos. The embryos were fixed for $10 \mathrm{~min}$ in $1 \%$ PFA prior to dissection of the dorsal skin. The epidermal and dermal layers were separated under a dissection microscope. Dissected back skins were permeabilized overnight $(0.5 \%$ Triton X$100,0.01 \%$ sodium deoxycholate, $1 \%$ bovine serum albumin, $0.02 \%$ sodium azide) prior to whole-mount immunostaining with CD31. To systematically analyze the same region for each embryo, 1 rectangular confocal image $(1,700 \times 1,100 \mu \mathrm{m})$ was taken at the anterior side of the skin specimen with the upper longer side of the rectangle placed on the midline. Within each rectangular picture the number of branch points was determined with the cell counter tool in ImageJ in 6 ROIs $(250 \times 250 \mu \mathrm{m}), 3$ in the top half and 3 in the bottom half of the rectangle, not overlapping with the larger arteries and veins. NEONATAL RETINAL ANGIOGENESIS: EC-specific GS deletion was obtained by IP administration of tamoxifen (Sigma; $10 \mathrm{mg} \mathrm{kg}^{-1}$; dissolved in 1:10 EtOH:oil solution) once daily from $\mathrm{P} 1$ to $\mathrm{P} 3$ in $\mathrm{GS}^{\mathrm{VECKO}}$ or once at P2 for $\mathrm{GS}^{\mathrm{pECKO}}$. For in vivo proliferation quantification, EdU (5ethynyl-2'-deoxyuridine; Invitrogen) was injected IP $2 \mathrm{~h}$ before sacrifice. Unless stated 
1137 otherwise, retinas were isolated at P5 as previously described ${ }^{41}$ and fixed in $2 \%$ PFA for 2

1138 h. Isolectin B4 (IB4), EdU, NG2 and CollV stainings were performed as previously 1139 described ${ }^{1,2}$. Radial outgrowth of the vascular plexus, vascular area, branch points, 1140 number of filopodia and number of distal sprouts were analysed on isolectin IB4-stained 1141 retinas (see below) with Image J. Numbers of branch points and EdU ${ }^{+}$ECs were quantified 1142 in $200 \times 200 \mu \mathrm{m}$ ROls; per retina 12 ROls were placed at the front of the vascular plexus 1143 and 8 ROls were placed more towards the center of the plexus. Filopodia and distal 1144 sprouts were quantified on ten high magnification (63x) images per retina, each 1145 representing approximately $200 \mu \mathrm{m}$ of utmost vascular front. For analysis of the retinal 1146 vasculature at P21 (3 week-old) and P42 (6 week-old) mice underwent the same 1147 tamoxifen treatment regimen as for analyses at P5. In addition, different tissues were 1148 collected from P42 mice for endoglin and CD34 staining to study blood vessels in different 1149 vascular beds. OXYGEN INDUCED RETINOPATHY: Oxygen induced retinopathy (ROP) was 1150 induced by exposing C57BL/6 pups to 70\% oxygen from P7-P12. Pups were then returned 1151 to normoxia and injected daily with $20 \mathrm{mg} \mathrm{kg}^{-1} \mathrm{MSO}$. At P17, pups were euthanized and 1152 eyes were enucleated, fixed in 4\% PFA and retinal flatmounts were stained for isolectin B4 1153 2,3. MSO-treated animals retained normal behavior notwithstanding observable weight 1154 loss. Mosaic tile images were captured using the inverted Leica DMI6000B 1155 epifluorescence microscope (Leica, Manheim, Germany) and analysis of the vascular tuft 1156 area (the complete retina was analyzed, no ROls were used) and the vaso-obliterated 1157 area was performed with NIH Image $\mathrm{J}$ software and are expressed as percentage of the 1158 total retinal area. CORNEAL (MICRO-)POCKET ASSAY (CPA) to induce neovascularization of 1159 the avascular cornea was performed as previously described ${ }^{42}$. In brief, in the eyes of 8 1160 week-old C57BL/6 mice, a lamellar micropocket was dissected toward the temporal limbus 1161 to allow placing of a basic fibroblast growth factor (bFGF)-containing pellet on the corneal 1162 surface. Five days after implanting the pellets, the mice were sacrificed, the eyes were 
1163

1164

1165

1166

1167

1168

1169

1170

1171

1172

1173

1174

1175

1176

1177

1178

1179

1180

1181

1182

1183

1184

1185

1186

1187

1188

enucleated and the corneas were excised and fixed in 70\% ethanol prior to CD31 antibody staining. After staining, the corneas were flat-mounted and imaged on a Zeiss LSM 780 confocal microscope. $\mathrm{CD} 31^{+}$area was measured in ImageJ after thresholding the signal and is expressed as \% of total cornea area. Production of the pellets was done as previously described ${ }^{42}$. The pellets contained $20 \mathrm{ng}$ bFGF and the concentration of MSO in the initial solution from which the pellets were made was $10 \mathrm{mM}$. IMIQUIMOD-INDUCED SKIN INFLAMMATION: Ten week old female Balb/C mice received a daily topical dose of $5 \%$ imiquimod cream $(62.5 \mathrm{mg})$ on their shaved backs for four days to induce skin inflammation ${ }^{3} .1 \mathrm{~h}$ after each administration of the cream, the same skin area was treated either with Vaseline ${ }^{\circledR}$ jelly or Vaseline ${ }^{\circledR}$ jelly containing MSO (low dose: $20 \mathrm{mg} \mathrm{kg}^{-1}$; or high dose: $40 \mathrm{mg} \mathrm{kg}^{-1}$ ). The MSO treatment did not affect bodyweight of the mice. Skins and spleens were collected and fixed in 4\% PFA. Paraffin sections of skins were stained for CD105 (R\&D Systems) and H\&E. Images were captured with a Leica DMI6000B microscope (Leica microsystems, Mannheim, Germany). Per animal, ten images representing different locations along the total length of the skin specimen were analyzed for $\mathrm{CD}_{105^{+}}$area. MILES VASCULAR PERMEABILITY ASSAY: 8 week old female Balb/C mice were treated for 3 consecutive days with $20 \mathrm{mg} \mathrm{kg}^{-1}$ day $^{-1} \mathrm{MSO}$ or with vehicle prior to injection with $300 \mu \mathrm{l} 0.5 \%$ Evan's blue dye. The inflammatory irritant mustard oil $(0.25 \mathrm{ml}$ allyl isothiocyanate in $4.75 \mathrm{ml}$ mineral oil) was applied on one of the ears with a cotton swab to induce vascular permeability. Mineral oil as a control was applied on the other ear. After $15 \mathrm{~min}$, again mustard oil/mineral oil was applied on the ear for $30 \mathrm{~min}$, after which the circulation was flushed with saline for 3 min and mice were perfused with $1 \%$ PFA in $50 \mathrm{mM}$ citrate buffer $(\mathrm{pH}=3.5)$ for $2 \mathrm{~min}$. Ears were cut and minced in formamide and incubated at $55{ }^{\circ} \mathrm{C}$ overnight to extract the Evan's blue from the tissue. Quantification of the dye was performed by a spectrophotometrical optical density measurement at $620 \mathrm{~nm}$. HeMATOLOGICAL PROFILING IN 6 WEEK-OLD MICE was performed with a Cell Dyn 3700 device 
1189 (Abbott Diagnostics) according to the manufacturer's guidelines. Plasma measurements

1190 for different liver/inflammation parameters were performed in the clinical laboratory of the 1191 university hospital of Leuven. Prior randomization was not applicable for any of the above 1192 mouse models given that all animal treatments were done in baseline conditions. No 1193 statistical methods were used to predetermine the sample size. For all mouse 1194 experiments, data analysis was done by researchers blinded to the group allocation. All 1195 animal procedures were approved by the Institutional Animal Care and Research Advisory 1196 Committee of the University of Leuven.

1197 IN SILICO SCREENING FOR PALMITOYLATION SITES: The human RHOJ protein sequence was 1198 screened for putative palmitoylation sites on the SwissPalm website ${ }^{22}$ entering 'RHOJ' as 1199 the protein name.

1200 MOdELING AND SIMULATIONS: The GS models were built starting from X-ray crystallographic 1201 structures retrieved from the Protein Data Bank (entry 2OJW for human GS and 1FPY for 1202 bacterial GS). All simulations were run with Gromacs $5.1 .4^{43}$ and the Amber FF14SB ${ }^{44}$ 1203 force field, while palmitoyl-CoA was parametrized with GAFF and the point charges were 1204 calculated with Gaussian $09^{45}$ at the Hartree-Fock level with a $6-31 G^{*}$ basis set. The 1205 different models were then embedded in a TIP3P water box, counter ions were added to 1206 ensure the overall charge neutrality. An initial 2,000 steps of steepest descent and 500 1207 steps of conjugated gradient were applied to minimize the geometry and remove steric 1208 clashes, followed by 10 ns of isothermal-isobaric (NPT) equilibration. The Berendsen 1209 barostat was applied to keep the pressure around $1 \mathrm{~atm}$, while the temperature of $300 \mathrm{~K}$ 1210 was maintained throughout all the simulations with the $\mathrm{V}$-rescale algorithm ${ }^{46} .500 \mathrm{~ns}$ long 1211 molecular dynamics production runs were carried out for all the systems in the canonical 1212 (NVT) ensemble, for a cumulative total of $2.5 \mu \mathrm{s}$. The particle mesh Ewald (PME)-Switch 1213 algorithm was used for electrostatic interactions with a cut-off of $1 \mathrm{~nm}$, and a single cut-off 
1214 of $1.2 \mathrm{~nm}$ was used for Van der Waals interactions. Four simulations for human GS and 1215 two for Salmonella typhimurium's GS were run by placing the CoA moiety close to the 1216 adenosine binding site and allowing different initial positions for the palmitoyl tail. The CoA 1217 head invariably docked and remained tightly bound to the adenine binding site in all 1218 simulations. Among these, two favorable alternative arrangements (Extended Data Fig. $8 b)$ for the tail were identified in both systems. In one of these conformations, the beginning of the palmitate tail (from the point of view of the CoA moiety) approaches very 1221 closely the conserved CYS209 (human residue numbering, Conformation A in Extended Data Fig. 8b, details in Extended Data Fig. 8c), and in the other conformation 1223 (Conformation B in Extended Data Fig. 8b, details in Extended Data Fig. 8d) it approaches 1224 the conserved Ser65 and 75.

1225 Multiple SEQUenCE ALIGNMENTS: A multiple sequence alignment of the GS protein across 1226 different species was performed with the Basic Local Alignment Search Tool (BLAST). The 1227 algorithm matches sequences according to local similarity, by optimizing their Maximal 1228 Segment Pair score (MSP). The 100 matches with the highest identity to the Homo 1229 sapiens sequence surrounding amino acid C209 were taken from the UniProtKB/Swiss1230 Prot refined database.

1231 Statistical ANALysis: Data represent mean \pm s.e.m. of pooled experiments unless 1232 otherwise stated. Scatters in bar graphs represent the values of independent experiments 1233 or individual mice. In case individual values are highly alike, scatter points overlap and 1234 may no longer be visible as individual points. $n$ values represent the number of 1235 independent experiments performed or the number of individual mice phenotyped. 1236 Statistical significance between groups was calculated with one of the following methods. 1237 For comparisons to point-normalized data, a two-tailed one-sample t-test was used in 1238 GraphPad Prism7. For pairwise comparisons, two-tailed unpaired $t$-tests were used in 
1239 GraphPad Prism7. For multiple comparisons within one data set, one-way ANOVA with

1240 Dunnett's multiple comparison (comparing every mean with the control mean rather than 1241 comparing every mean with every other mean) was used in GraphPad Prism7. Mixed 1242 model statistics (this test does not assume normality or equal variance) was used with the 1243 experiment as random factor only in case confounding variation in baseline measurements 1244 between individual EC isolations (for each experiment, ECs were freshly isolated from 1245 individual human umbilicals) or mouse litters precluded the use of the above described 1246 statistical tests. For this, R and the Ime4 package were used; $P$ values were obtained with 1247 the Kenward-Roger F-test for small mixed effect model datasets. In the most severe 1248 cases, the individual datapoints (each datapoint being the mean of the technical replicates 1249 within an experiment or an individual animal) in the bar graphs have been color-coded per 1250 experiment or per litter to show the baseline variation. Sample size for each experiment 1251 was not pre-determined. A $P$ value $<0.05$ was considered significant.

1252 Data avallability: Fig.1, Fig. 4, Fig. 5, Extended Data Fig. 1, Extended Data Fig. 7 and 1253 Extended Data Fig. 8 have associated raw data (uncropped blots and/or gel pictures) in 1254 Supplemental Information Fig. 1. Fig. 1, Fig. 2, Extended Data Fig. 1 and Extended Data 1255 Fig. 4 have associated raw data (Excel files) for all bar graphs representing data from 1256 experiments involving mouse models. For the molecular modelling of palmitoyl-CoA 1257 docking into GS, models and trajectories are available on Figshare (doi: 1258 10.6084/m9.figshare.6575438). Any additional information required to interpret, replicate 1259 or build upon the Methods or findings reported in the manuscript is available from the 1260 corresponding author upon request. 
1264

1265

1266

1267

1268

1269

1270

1271

1272

1273

1274

1275

1276

1277

1278

1279

1280

1281

1282

1283

1284

1285

1286

1287

1288

1289

1290

1291

1292

1293

1294

1295

1296

1297

1298

1299

1300

25 Zheng, B., Zhu, S. \& Wu, X. Clickable analogue of cerulenin as chemical probe to explore protein palmitoylation. ACS Chem Biol 10, 115-121, doi:10.1021/cb500758s (2015).

26 Martin-Ramirez, J., Hofman, M., van den Biggelaar, M., Hebbel, R. P. \& Voorberg, J. Establishment of outgrowth endothelial cells from peripheral blood. Nat Protoc 7, 1709-1715, doi:10.1038/nprot.2012.093 (2012).

27 Van Den Bosch, L., Vandenberghe, W., Klaassen, H., Van Houtte, E. \& Robberecht, W. $\mathrm{Ca}(2+)$-permeable AMPA receptors and selective vulnerability of motor neurons. J Neurol Sci 180, 29-34 (2000).

28 McKinney, S. A., Murphy, C. S., Hazelwood, K. L., Davidson, M. W. \& Looger, L. L. A bright and photostable photoconvertible fluorescent protein. Nat Methods 6, 131133, doi:10.1038/nmeth.1296 (2009).

29 Hamel, L. D., Deschenes, R. J. \& Mitchell, D. A. A fluorescence-based assay to monitor autopalmitoylation of zDHHC proteins applicable to high-throughput screening. Anal Biochem 460, 1-8, doi:10.1016/j.ab.2014.05.013 (2014).

30 Kummel, D., Heinemann, U. \& Veit, M. Unique self-palmitoylation activity of the transport protein particle component Bet3: a mechanism required for protein stability. Proc Natl Acad Sci U S A 103, 12701-12706, doi:10.1073/pnas.0603513103 (2006).

31 Fraccaroli, A. et al. Endothelial alpha-parvin controls integrity of developing vasculature and is required for maintenance of cell-cell junctions. Circulation research 117, 29-40, doi:10.1161/CIRCRESAHA.117.305818 (2015).

32 Ho, C. Y., Jaalouk, D. E., Vartiainen, M. K. \& Lammerding, J. Lamin A/C and emerin regulate MKL1-SRF activity by modulating actin dynamics. Nature 497, 507-511, doi:10.1038/nature12105 (2013).

33 Timmerman, I. et al. A local VE-cadherin and Trio-based signaling complex stabilizes endothelial junctions through Rac1. J Cell Sci 128, 3041-3054, doi:10.1242/jcs.168674 (2015).

34 van Buul, J. D. et al. RhoG regulates endothelial apical cup assembly downstream from ICAM1 engagement and is involved in leukocyte trans-endothelial migration. $J$ Cell Biol 178, 1279-1293, doi:10.1083/jcb.200612053 (2007).

35 Heemskerk, N. et al. F-actin-rich contractile endothelial pores prevent vascular leakage during leukocyte diapedesis through local RhoA signalling. Nat Commun $\mathbf{7}$, 10493, doi:10.1038/ncomms10493 (2016).

36 Dedecker, P., Duwe, S., Neely, R. K. \& Zhang, J. Localizer: fast, accurate, opensource, and modular software package for superresolution microscopy. $J$ Biomed Opt 17, 126008, doi:10.1117/1.JBO.17.12.126008 (2012). 
1301

1302

1303

1304

1305

1306

1307

1308

1309

1310

1311

1312

1313

1314

1315

1316

1317

1318

1319

1320

1321

1322

1323

1324

1325

1326

1327

1328

1329

1330

1331

1332

37 Persson, F., Linden, M., Unoson, C. \& Elf, J. Extracting intracellular diffusive states and transition rates from single-molecule tracking data. Nat Methods 10, 265-269, doi:10.1038/nmeth.2367 (2013).

$38 \mathrm{He}, \mathrm{Y}$. et al. Glutamine synthetase deficiency in murine astrocytes results in neonatal death. Glia 58, 741-754, doi:10.1002/glia.20960 (2010).

39 Benedito, R. et al. The notch ligands DII4 and Jagged1 have opposing effects on angiogenesis. Cell 137, 1124-1135, doi:S0092-8674(09)00324-9 [pii]

10.1016/j.cell.2009.03.025 (2009).

40 Claxton, S. et al. Efficient, inducible Cre-recombinase activation in vascular endothelium. Genesis 46, 74-80, doi:10.1002/dvg.20367 (2008).

41 Pitulescu, M. E., Schmidt, I., Benedito, R. \& Adams, R. H. Inducible gene targeting in the neonatal vasculature and analysis of retinal angiogenesis in mice. Nat Protoc 5, 1518-1534, doi:10.1038/nprot.2010.113 (2010).

42 Kenyon, B. M. et al. A model of angiogenesis in the mouse cornea. Invest Ophthalmol Vis Sci 37, 1625-1632 (1996).

43 Abraham, M. J. et al. GROMACS: High performance molecular simulations through multi-level parallelism from laptops to supercomputers. SoftwareX 1, 19-25 (2015).

44 Maier, J. A. et al. ff14SB: Improving the Accuracy of Protein Side Chain and Backbone Parameters from ff99SB. J Chem Theory Comput 11, 3696-3713, doi:10.1021/acs.jctc.5b00255 (2015).

45 Frisch, M. et al. Gaussian 09, Revision C.01. Gaussian Inc., Wallingford CT. (2009).

46 Bussi, G., Donadio, D. \& Parrinello, M. Canonical sampling through velocity rescaling. J Chem Phys 126, doi:Artn 014101

10.1063/1.2408420 (2007). 
1350 Extended Data table 1: Weight, hematological and Blood plasma 1351 PARAMETERS FOR 6 WEEK-OLD GS ${ }^{\mathrm{VECKO}}$ MICE AND CONTROL LITTERMATES

1352 Values are mean \pm s.e.m. of $\mathrm{n}=14$ (control) vs $\mathrm{n}=17\left(\mathrm{GS}^{\mathrm{VECKO}}\right)$ animals. ${ }^{*} P=0.0232$ vs 1353 control, Student's $t$ test. WBCs, white blood cells; RBCs, red blood cells, AST, aspartate 1354 amino transferase; ALT, alanine amino transferase; $\gamma$-GT, gamma-glutamyl transferase; 1355 CRP; C-reactive protein. 


\section{Extended Data table 2: Alignment of the amino acid sequence} ENCOMPASSING THE C209 RESIDUE ACROSS DIFFERENT SPECIES.

1359

1360

1361

1362

1363

1364

1365

1366

1367

1368

1369

1370

1371

1372

1373

1374

1375

1376

1377

1378

1379

1380

1381

Multiple sequence alignment showing the conservation of amino acid C209 (in red) in GS across different species. Here the sequence alignment of 41 residues surrounding this cysteine is shown for up to 100 of the closest sequence identity matches with Homo sapiens GS obtained with BLAST from the UniProtKB/Swiss-Prot database. C209 is mostly conserved across species and when not conserved it is often substituted by residues (Ser or Thr) that can (in theory) be palmitoylated as well. In Escherichia coli (shown at the bottom), for example, a Thr is found at the structurally equivalent position (T210, highlighted in yellow). If for one and the same species multiple GS isoforms are known, only the one with the highest \% identity is shown.

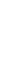


and mouse astrocytes (c) (representative immunoblots of two independent experiments 1383 are shown). d-e, Genomic organization of the loxed GS allele before and after Cremediated excision (d) and correct recombination of the lox allele (L) in GS ${ }^{\mathrm{vECKO}}$ and $\mathrm{GS}^{\mathrm{pECKO}}$ mice upon tamoxifen (tam) treatment, as assessed by genomic DNA PCR (e; the PCR to amplify the loxed GS allele (lox) or to amplify the Cre-recombined allele $(\Delta)$ were run in separate reactions but loaded in the same lane; the gel picture shown is representative for all control, vECKO and pECKO mice used in this study). f, Quantification of branchpoints at the rear of the plexus in GS ${ }^{\mathrm{VECKO}}$ mice (mean \pm s.e.m.; $n=10$ animals for $\mathrm{GS}^{\mathrm{vECKO}}$ and 11 for wild-type (WT) controls from 3 litters; ${ }^{\star} P<0.05$ vs WT littermates, mixed models $\mathrm{R}$ statistics). $\mathbf{g}$, Pericyte coverage of retinal microvessels in WT and GS ${ }^{\mathrm{vECKO}}$ littermates determined by NG2 staining and shown as $\mathrm{NG}^{+}$area as \% of vessel area (mean \pm s.e.m.; $n=4$ animals for WT and 3 for $\mathrm{GS}^{\mathrm{vECKO}}$ from 1 litter; ${ }^{\mathrm{NS}} P>0.05$ vs WT,

Student's $t$ test). $\mathbf{h}$, Reduced complexity of the retinal vascular front in P5 GS ${ }^{\mathrm{vECKO}}$ vs WT

animals determined by the number of branches on distal sprouts (mean \pm s.e.m.; $n=13$ animals for WT and 21 for GS ${ }^{\mathrm{vECKO}}$ from 5 litters; ${ }^{*} P<0.05$ vs WT, Student's $t$ test). i,

Quantification of EdU ${ }^{+}$ECs at the rear of the plexus (mean \pm s.e.m.; $n=12$ animals for WT and 22 for $\mathrm{GS}^{\mathrm{vECKO}}$ from 4 litters; ${ }^{\mathrm{NS}} \mathrm{P}>0.05$ vs WT littermates, Student's $t$ test). j-m, Isolectin B4 staining of P5 retinal vascular plexi from WT (representative pictures with zoom-in insets, $A=$ artery, $V=$ vein) and quantification of branch points at the front $(I)$ and the rear $(m)$ of the plexus (mean \pm s.e.m.; $n=10$ animals for WT and 18 for $G S^{p E C K O}$ from 4 litters; ${ }^{*} P \leq 0.05$ vs WT littermates, Student's $t$ test). $\mathbf{n}-\mathbf{u}$, 1403 Isolectin B4 staining of the retinal microvasculature of 3 week (P21)-old $(\mathrm{n}, \mathrm{o})$ and 6 week (P42)-old $(r, s)$ WT and GS ${ }^{\text {vECKO }}$ littermates ( $A=$ artery, $V=$ vein). Lower left insets display higher magnification of IB4-stained superficial plexus, whereas lower right insets display higher magnification of the deep plexus. Also shown is the corresponding quantification of 1407 the vascular area $(p, t)$ and the branch point density $(q, u)$ in the superficial and the deep 


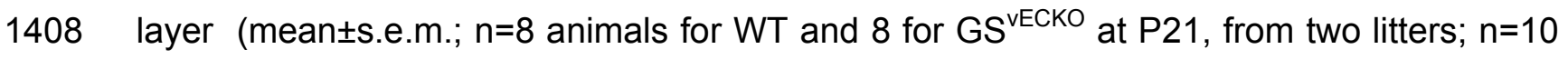
1409 animals for WT and 14 for $\mathrm{GS}^{\mathrm{VECKO}}$ at P42, from four litters; ${ }^{\mathrm{NS}} P>0.05$ vs WT, Student's $t$ 1410 test). v-ag, Representative micrographs of heart $(v, z)$, liver ( $w, a a)$ and kidney $(x, a b)$ 1411 sections from WT and GS ${ }^{\mathrm{VECKO}}$ littermates immunostained for the EC marker endoglin and 1412 of lung $(\mathrm{y}, \mathrm{ac})$ sections immunostained for the EC marker CD34 and corresponding 1413 quantifications of endoglin ${ }^{+}$(ad, heart; ae, liver; af, kidney) or CD34 ${ }^{+}$(ag) vascular area

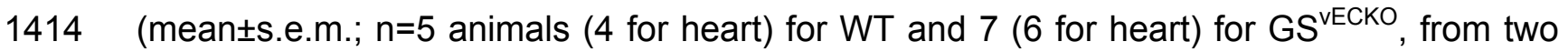
1415 litters, ${ }^{N S} P>0.05$ vs WT, Student's $t$ test). ah-ai, Images of flat-mounted retinas from 1416 control (ah) and MSO-treated (ai) ROP mice (vaso-obliterated area in white). Images 1417 shown are representative for 7 (ah) and 6 (ai) mice. Exact $P$ values: (a) HUVEC vs lung 1418 ECs: 0.0278; HUVEC vs colon ECs: 0.1086; HUVEC vs liver ECs: 0.3334; HUVEC vs 1419 HEPG2: <0.0001; (f) <0.0001; (g) 0.3491; (h) <0.0001; (i) 0.8247; (l) 0.0012; (m) 0.050; (p) 1420 superficial: 0.1218; deep: 0.1720; (q) superficial: 0.9995; deep: 0.4289; (t) superficial: 1421 0.9792; deep: 0.6602; (u) superficial: 0.7979; deep: 0.1275; (ad) 0.9021; (ae) 0.2279; (af) 14220.7647 ; (ag) 0.3614 . Scale bars are $200 \mu \mathrm{m}$ in j-k, n-o and r-s, $20 \mu \mathrm{m}$ in v-ac and $1 \mathrm{~mm}$ in 1423 ah-ai. HEPG2: hepatocellular carcinoma cells; mLiEC: mouse liver ECs; Tam: tamoxifen; 1424 lox: loxed allele; $\Delta$ : recombined allele; NG2: chondroitin sulfate proteoglycan 4; Edu: 51425 ethynyl-2'-deoxyuridine. For gel source images, see Supplemental Information Fig. 1.

1427 Extended DATA FIgURE 2: EfFECTS OF SILENCING AND PHARMACOLOGICAL INHIBITION 1428 OF GS ON EC VIABILITY AND CENTRAL METABOLISM

1429 a, GS mRNA levels in control ECs and ECs transduced with two different non-overlapping 1430 shRNAs targeting $G S\left(G S^{K D 1}\right.$ and $G S^{K D 2} ; G^{K D 1}$ is used in the experiments in the main 1431 manuscript and denoted as $\mathrm{GS}^{\mathrm{KD}}$ ) or transfected with scrambled siRNA (SCR) or siRNA 1432 targeting GS (siGS). Data are expressed as \% of the respective control, denoted by the

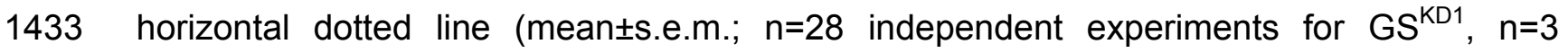


1434 independent experiments for $G S^{K D 2}$ and $n=9$ independent experiments for siGS; * $P<0.05$ 1435 vs the respective control; one sample $t$ test). b-c, Quantification of number of sprouts (b) 1436 and total sprout length (c) for spheroid sprouting assays with $\mathrm{GS}^{\mathrm{KD}} \mathrm{ECs}$ and $G S^{\mathrm{KD}} \mathrm{ECs}$ 1437 expressing a shRNA-resistant GS mutant ( $\mathrm{GS}^{\mathrm{OE}}$ ) (mean \pm s.e.m.; $\mathrm{n}=3$ independent 1438 experiments; ${ }^{*} P<0.05$ and ${ }^{N S} P>0.05$ vs control; ANOVA with Dunnett's multiple 1439 comparison vs control). d, Viability of control and GS ${ }^{\mathrm{KD}} \mathrm{ECs}$ as measured by lactate 1440 dehydrogenase (LDH) release assay (meanıs.e.m.; n=3 independent experiments; $1441{ }^{\mathrm{NS}} \mathrm{P}>0.05$ vs control, one sample $t$ test). e, Intracellular reactive oxygen species (ROS) 1442

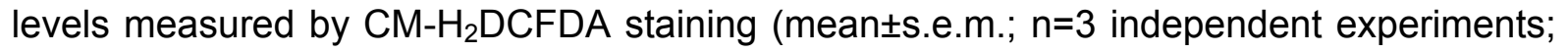
${ }^{N S} P>0.05$ vs control, Student's $t$ test). f, Energy charge measurement (([ATP] + V2[ADP]) / 1444 $([A T P]+[A D P]+[A M P]))$ in $G^{K D}$ and control ECs (mean \pm s.e.m.; $n=3$ independent 1445 experiments; ${ }^{N S} P>0.05$ vs control, Student's $t$ test). g, Ratio of oxidized glutathione over 1446

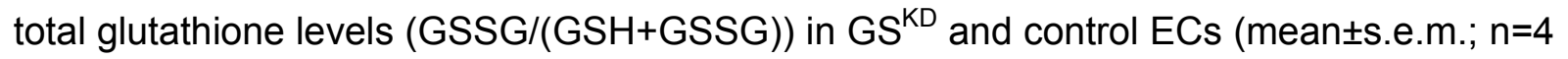
1447 independent experiments; ${ }^{N S} P>0.05$ vs control, Student's $t$ test). $\mathbf{h}$, NADP/NADPH ratio in $1448 \mathrm{GS}^{\mathrm{KD}}$ and control ECs (mean \pm s.e.m.; $\mathrm{n}=5$ independent experiments; ${ }^{\mathrm{NS}} \mathrm{P}>0.05$ vs control, 1449 one sample $t$ test). i-k, Effect of $G S^{K D}$ on major metabolic fluxes including glycolysis (i), 1450 glucose oxidation (j) and glutamine oxidation (k) (mean \pm s.e.m.; $n=3$ independent 1451 experiments for (i), $\mathrm{n}=5$ for (j) and $\mathrm{n}=4$ for (k); ${ }^{\mathrm{NS}} \mathrm{P}>0.05$ vs control, one sample $t$ test). I,m, 1452 Oxygen consumption rate (OCR) in control, MSO-treated and GS ${ }^{\mathrm{KD}}$ ECs in basal state and 1453 after injection of oligomycin, FCCP and antimycin A (I) (meanıs.e.m.; n=3 independent 1454 experiments), and calculation of $\mathrm{OCR}_{\mathrm{BAS}}$, OCR $\mathrm{OCTP}_{\mathrm{ATP}}$ and maximal respiration $(\mathrm{m})$

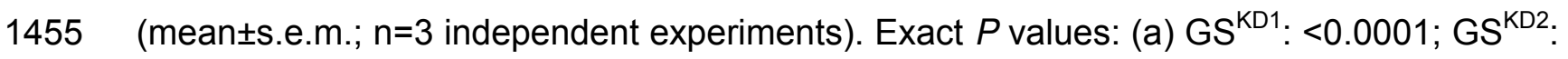
$1456<0.0001$; siGS: <0.0001; (b) ctrl vs GS ${ }^{\mathrm{KD}}: 0.0147$; $\operatorname{ctrl} v s \mathrm{GS}^{\mathrm{KD}}+\mathrm{rGS}^{\mathrm{OE}}: 0.9824$; (c) ctrl vs 1457 GS ${ }^{K D}: 0.0083$; ctrl vs $\mathrm{GS}^{\mathrm{KD}}+\mathrm{rGS}^{\mathrm{OE}}$ : 0.6528; (d) 0.5717; (e) 0.8206 ; (f) 0.3715; (g) 0.4398; 1458 (h) 0.9291 ; (i) 0.4691 (j) 0.6643 (k) 0.6786. AU: arbitrary units; CM-DCF: 5-(and-6)1459 chloromethyl-2',7'-dichlorodihydrofluorescein diacetate, acetyl ester; FCCP: carbonyl 
cyanide-4-(trifluoromethoxy) phenylhydrazone; $\mathrm{OCR}_{\mathrm{BAS}}$ : basal oxygen consumption rate;

1461 OCR $_{\text {ATP: }}$ ATP-generating oxygen consumption rate; RFU: relative fluorescence units; $1462 \mathrm{MSO}$, methionine sulfoximine.

\section{EXTENDEd DATA FIgURE 3: GS KNOCK-DOWN REDUCES EC MOTILITY}

1465 a, Wound closure in control and $\mathrm{GS}^{\mathrm{KD} 2} \mathrm{EC}$ monolayer scratch assays with or without 1466 MitoC-pretreatment (mean \pm s.e.m.; $\mathrm{n}=7$ and 5 independent experiments for with and 1467 without MitoC respectively; ${ }^{*} P<0.05$ vs corresponding control; Student's $t$ test). b,

1468 Quantification of lamellipodial area (\% of total cellular area) in control and GS ${ }^{\text {KD2 }}$ ECs 1469 (mean \pm s.e.m.; $\mathrm{n}=3$ independent experiments; ${ }^{*} P<0.05$ vs control; Student's $t$ test). c, 1470 Wound closure in monolayer scratch assays with SCR- and siGS-transfected ECs 1471 (mean \pm s.e.m.; $\mathrm{n}=5$ independent experiments; ${ }^{*} P<0.05$ vs SCR; Student's $t$ test). d, 1472 Quantification of lamellipodial area (\% of total cellular area) in SCR- and siGS-transfected 1473 ECs (mean \pm s.e.m.; $n=5$ independent experiments; ${ }^{*} P<0.05$ vs SCR; Student's $t$ test). e, $1474\left[{ }^{3} \mathrm{H}\right]$-Thymidine incorporation into DNA in SCR- and siGS-transfected ECs (mean \pm s.e.m.; $1475 \mathrm{n}=3$ independent experiments; ${ }^{N S} P>0.05$ vs SCR; Student's $t$ test). Exact $P$ values: (a) ctrl 1476 vs GS ${ }^{\mathrm{KD} 2}$ : 0.0290; $\operatorname{ctrl}$ vs GS ${ }^{\mathrm{KD} 2}+$ MitoC: 0.0223; (b) 0.0088; (c) 0.0407; (d) 0.0083; (e) 14770.4335.

1479 Extended Data Figure 4: Effects of GS Silencing ON CYTOSKeleton AND 1480 BARRIER FUNCTION

1481 a-h, Images of control $(a, c, e, g)$ and $G^{K D}$ (b,d,f,h) ECs after staining for $\alpha$-tubulin $(a, b)$, F1482 actin $(\mathrm{c}, \mathrm{d})$ and nuclear staining $(\mathrm{e}, \mathrm{f})$; images shown are representative for 3 independent 1483 experiments. i-k, Representative images of phalloidin + Hoechst-stained liver ECs 6 hours 1484 after isolation from control (i) and MSO-treated (j) mice, and corresponding quantification 
1485 of F-actin levels (k) (mean \pm s.e.m.; $n=5$ mice per group; ${ }^{*} P<0.05$ vs control, Student's $t$ 1486 test). I-n, Representative images of phalloidin-stained (F-actin) confluent monolayer 1487 control $(\mathrm{I})$ and $\mathrm{GS}^{\mathrm{KD}}(\mathrm{m})$ ECs aligning a scratch wound, and quantification of F-actin levels 1488 (n) (mean \pm s.e.m.; $n=5$ independent experiments; ${ }^{*} P<0.05$ vs control, Student's $t$ test). $\mathbf{0}$, 1489 Quantification of the length of discontinuous and continuous VE-cadherin-stained junctions 1490 in control and GS ${ }^{K D}$ ECs (mean \pm s.e.m.; $\mathrm{n}=4$ independent experiments; ${ }^{*} P<0.05$ vs control, 1491 Student's $t$ test). p, Quantification of VE-cadherin gap size index in control and GS ${ }^{\mathrm{KD}} \mathrm{EC}$ 1492 monolayers (mean \pm s.e.m.; $\mathrm{n}=4$ independent experiments; ${ }^{*} P<0.05$ vs control, Student's $t$ 1493 test). $\mathbf{q - v}$, Corresponding representative images of monolayer control and GS ${ }^{\mathrm{KD}} \mathrm{ECs}$ 1494 stained for VE-cadherin $(q, r, u, v)$ and F-actin $(s, t, u, v)$. Yellow arrows in ( $r$ ) point to 1495 discontinuous VE-cadherin junctions and yellow asterisks indicate intracellular gaps. w, 1496 Quantification of transendothelial electrical resistance (TEER) in control and GS ${ }^{\mathrm{KD}} \mathrm{EC}$ 1497 monolayers (mean \pm s.e.m.; $\mathrm{n}=4$ independent experiments; ${ }^{*} P<0.05$ vs control, Student's $t$ 1498 test at each time point). $\mathbf{x - z}$, Quantification (x) of Evans blue dye extracted from the ears of 1499 control and MSO-treated mice induced by topical application of mustard oil ( $\mathrm{n}=4$ mice for 1500 each condition, ${ }^{\star} P<0.05$; Student's $t$ test) and representative pictures of the Evans blue 1501 leakage into the ear tissue in control $(\mathrm{y})$ and MSO-treated $(\mathrm{z})$ mice. Exact $P$ values: $(\mathrm{k})$ 1502 0.0030; (n) 0.0036; (o) continuous ctrl vs GS ${ }^{\mathrm{KD}}$ : 0.0005; discontinuous ctrl vs GS ${ }^{\mathrm{KD}}$ : 15030.0005 ; (p) 0.0356; (w) 0.0181; (x) 0.0002. Scale bar is $20 \mu \mathrm{m}$ in a-h and in I-m and $10 \mu \mathrm{m}$ 1504 in i-j and in q-v. AU: arbitrary units.

1506 EXTENDEd DATA FIgURE 5: ENZYMATIC ACTIVITY OF GS AND ITS ROLE IN EC MIGRATION 1507 a, Scheme of ${ }^{15} \mathrm{NH}_{4}{ }^{+}$labeling of glutamate and glutamine with unlabeled carbons (blue) 1508 and labeled nitrogens (red). b, ${ }^{15} \mathrm{~N}$ incorporation into glutamine (\% isotope enrichment in $1509 \mathrm{~m}+1$ and $\mathrm{m}+2,30 \mathrm{~min}$ after adding ${ }^{15} \mathrm{NH}_{4}{ }^{+}$) in medium with dialyzed serum and different 1510 glutamine concentrations (mean \pm s.e.m.; $n=3$ independent experiments; ANOVA with 
1511 Dunnett's multiple comparisons vs $4 \mathrm{mM}$; $\left.{ }^{*} P<0.05\right)$. c, ${ }^{15} \mathrm{~N}$ incorporation into glutamate $(\%$ 1512 isotope enrichment in $m+1$ ) and glutamine (\% isotope enrichment in $m+1$ and $m+2), 30$ 1513 min after adding increasing concentrations of ${ }^{15} \mathrm{NH}_{4} \mathrm{Cl}$ (mean \pm s.e.m.; $\mathrm{n}=3$ independent 1514 experiments). d, Scheme of glutamine labeling from $\left[\mathrm{U}-{ }^{13} \mathrm{C}\right]$-glutamate with unlabeled 1515 nitrogens (blue) and labeled carbons (red). e, Label contribution of $\left[\mathrm{U}-{ }^{13} \mathrm{C}\right]$-glutamate to 1516 intracellular glutamine at various glutamine concentrations (\% isotope enrichment in 1517 glutamine and glutamate $\mathrm{m}+5,30 \mathrm{~min}$ after adding the tracer) (mean \pm s.e.m.; $\mathrm{n}=3$ 1518 independent experiments; ANOVA with Dunnett's multiple comparisons vs $4 \mathrm{mM}$; $P$ 1519 <0.05). f, Scheme for $\left[\mathrm{U}-{ }^{13} \mathrm{C}\right]$-glucose carbon contribution to glutamine with labeled 1520 carbons (red) and unlabeled carbons (blue). Incorporation is shown after one turn of the 1521 TCA cycle. $\mathbf{g}$, Total contribution of $\left[\mathrm{U}-{ }^{13} \mathrm{C}\right]$-glucose carbons to $\alpha$-ketoglutarate, glutamate 1522 and glutamine in ECs in medium with or without glutamine, $48 \mathrm{~h}$ after adding the tracer

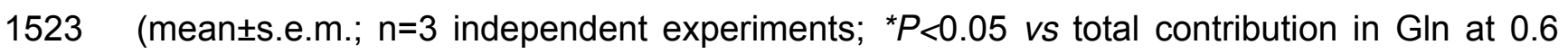
$1524 \mathrm{mM}$ external GIn, ANOVA with Dunnett's multiple comparisons). $\mathbf{h},{ }^{15} \mathrm{~N}$ incorporation into 1525 glutamine (\% isotope enrichment in $\mathrm{m}+1$ and $\mathrm{m}+2,30 \mathrm{~min}$ after adding ${ }^{15} \mathrm{NH}_{4}{ }^{+}$) in ECs and 1526 HEPG2 cells (mean \pm s.e.m.; $n=4$ independent experiments (ND=not detected)). i, ${ }^{13} \mathrm{C}-$ 1527 glutamine uptake kinetics in control, MSO-treated and GS ${ }^{K D}$ ECs and subsequent 1528 conversion to glutamate. See Methods for explanation of the different time points. Data are 1529 expressed as $\mathrm{m}+5$ isotopomer, as percentage of the total intracellular glutamine ( $\mathrm{gln}$ ) or 1530 glutamate (glu) pool (mean \pm s.e.m.; $n=3$ independent experiments, except for $30 \mathrm{~min}$ 1531 where $n=1$ experiment; no statistical differences between control, MSO-treated and GS ${ }^{K D}$ 1532 were observed for glutamine nor for glutamate; ANOVA with Dunnett's multiple 1533 comparison vs control at each time point; no statistical analysis was performed at $30 \mathrm{~min}$ ). $1534 \mathrm{j},{ }^{14} \mathrm{C}$-glutamine uptake in control and $\mathrm{GS}^{\mathrm{KD}}$ ECs (mean \pm s.e.m.; $\mathrm{n}=5$ independent 1535 experiments; ${ }^{N S} P>0.05$ vs control, one sample $t$ test). $\mathbf{k}$, Ratio of intracellular glutamine 1536 (GIn) over glutamate (Glu) levels in control and GS ${ }^{K D}$ ECs (mean \pm s.e.m.; $n=3$ independent 
1537 experiments; ${ }^{N S} P>0.05$ vs control, Student's $t$ test). I, Velocity measurement of control and $1538 \mathrm{GS}^{\mathrm{KD}} \mathrm{ECS}$ at different glutamine (GIn) concentrations (mean \pm s.e.m.; $\mathrm{n}=4$ independent 1539 experiments; ${ }^{*} P<0.05$ vs corresponding control, mixed models R statistics). m-n, Effect of 1540 glutamine concentration on sprout number $(m)$ and total sprout length $(n)$ in control and $1541 \mathrm{GS}^{\mathrm{KD}}$ spheroids (mean \pm s.e.m.; $\mathrm{n}=3$ independent experiments; * $P<0.05$ vs corresponding 1542 control, mixed models R statistics). o-p, Number of sprouts per spheroid (o) and total 1543 sprout length $(p)$ in control and MSO-treated EC spheroids (mean \pm s.e.m.; $n=3$ 1544 independent experiments; ${ }^{\star} P<0.05$ vs control, paired Student's $t$ test). q-s, Effect of MSO1545 treatment on EC motility parameters: wound closure of MitoC-treated ECs (q) 1546 (mean \pm s.e.m.; $\mathrm{n}=11$ independent experiments; ${ }^{*} P<0.05$ vs control, Student's $t$ test), 1547 lamellipodial area (r) (mean \pm s.e.m.; $n=10$ independent experiments; * $P<0.05$ vs control, 1548 paired Student's $t$ test) and F-actin levels, $1 \mathrm{~h}$ after latrunculin wash-out (s) (mean \pm s.e.m.; $1549 \mathrm{n}=4$ independent experiments; ${ }^{*} P<0.05$ vs control, one-sample $t$ test). $\mathbf{t},\left[{ }^{3} \mathrm{H}\right]$-Thymidine 1550 incorporation in control and MSO-treated ECs (mean \pm s.e.m.; $n=3$ independent 1551 experiments; ${ }^{N S} P>0.05$ vs control, one sample $t$ test). Exact $P$ values: (b) $\mathrm{m}+10.025 \mathrm{mM}$ 1552 vs m+1 4 mM: 0.0096; m+1 0.6 mM vs m+1 4 mM: 0.1206; m+2 0.025 mM vs m+2 4 mM: 1553 0.0839; m+2 0.6 mM vs m+2 4 mM: 0.9921; (e) Glu m+5 0.6 mM vs Glu m+5 4 mM: 1554 0.9372; Glu m+5 0.025 mM + MSO vs Glu m+5 4 mM: 0.0034; Glu m+5 0.025 mM vs Glu 1555 m+5 4 mM: 0.0215; Gln m+5 0.6 mM vs Gln m+5 4 mM: 0.9297; Gln m+5 0.025 mM + 1556 MSO vs Gln m+5 4 mM: 0.9961; Gln m+5 0.025 mM vs Gln m+5 4 mM: 0.0268; (g) $\alpha$-keto 15570.6 mM vs Gln 0.6 mM: 0.0001; Glu 0.6 mM vs Gln 0.6 mM: 0.0001; Gln 0 mM vs Gln 0.6 $1558 \mathrm{mM}$ : 0.0285; (i) Gln 0.5 min: ctrl vs MSO: 0.4846; ctrl vs GS ${ }^{\mathrm{KD}}$ : 0.5904; Gln 10 min: ctrl vs 1559 MSO: 0.6709; ctrl vs GS ${ }^{\mathrm{KD}}$ : 0.6910; GIn $20 \mathrm{~min}$ : ctrl vs MSO: 0.5896; ctrl vs GS ${ }^{\mathrm{KD}}$ : 0.6784; 1560 Glu 0.5 min: ctrl vs MSO: 0.9774; ctrl vs GS ${ }^{\mathrm{KD}}$ : 0.8810; Glu $10 \mathrm{~min}$ : ctrl vs MSO: 0.0502; 1561 ctrl vs GS ${ }^{\mathrm{KD}}$ : 0.9598; Glu $20 \mathrm{~min}$ : ctrl vs MSO: 0.9782; $\operatorname{ctrl}$ vs $\mathrm{GS}^{\mathrm{KD}}$ : 0.7783. (j) 0.6623; (k) 1562 0.6704; (I) ctrl vs GS ${ }^{\mathrm{KD}} 0.1 \mathrm{mM}: 0.0054$; ctrl vs $\mathrm{GS}^{\mathrm{KD}} 0.6 \mathrm{mM}: 0.0247 \mathrm{ctrl}$ vs $\mathrm{GS}^{\mathrm{KD}} 2 \mathrm{mM}$ : 
$15630.0017 ;(\mathrm{m}) \mathrm{ctrl} v s \mathrm{GS}^{\mathrm{KD}} 0.6 \mathrm{mM}$ and $10 \mathrm{mM}$ : < 0.0001; (n) ctrl vs $\mathrm{GS}^{\mathrm{KD}} 0.6 \mathrm{mM}$ and 10 1564 mM: <0.0001; (o) 0.0313; (p) 0.0075; (q) 0.0019; (r) 0.0116; (s) 0.0091; (t) 0.5110. $\alpha$-keto: $1565 \alpha$-ketoglutarate; GDH: glutamate dehydrogenase; Glu: glutamate; GS: glutamine 1566 synthetase; GIn: glutamine; MSO, methionine sulfoximine; MitoC: mitomycin C.

1568 Extended DATA Figure 6: RESCUING THE GS ${ }^{\mathrm{KD}}$ PHENOTYPE IN VITRO

1569 a, Schematic representation of the DORA RHOA FRET biosensor, depicting from N- to C1570 terminal the circular permutated RHOA effector protein kinase $\mathrm{N}$ (cpPKN), the dimeric 1571 circular permutated Venus (dcpVen), the ribosomal protein-based linkers (L9), the dimeric 1572 Cerulean3 (dCer3) and RHOA. b-m, Representative images of control (b-d), MSO-treated $1573(\mathrm{e}-\mathrm{g}), \mathrm{GS}^{\mathrm{KD}}(\mathrm{h}-\mathrm{j})$ and $\mathrm{RHOJ}^{\mathrm{KD}}(\mathrm{k}-\mathrm{m})$ ECs after staining for F-actin (phalloidin) 1574 (b,d,e,g,h,j,k,m) and pMLC (c,d,f,g,i,j,l,m). n, Quantification of the pMLC-immunoreactivity 1575 (mean \pm s.e.m.; $\mathrm{n}=5$ independent experiments; ${ }^{*} P<0.05$ vs control, one sample $t$ test). o-t, 1576 Representative images of control $(0, q, s)$ and $G S^{K D}(p, r, t) E C$ spheroids treated with vehicle 1577 (o,p) or the ROCK inhibitors Y27632 (q,r) or fasudil hydrochloride (s,t). u-v, Quantification 1578 of the number of sprouts per spheroid $(u)$ and sprout length (v) (mean \pm s.e.m.; $n=3$ 1579 independent experiments; ${ }^{*} P<0.05$ and ${ }^{N S} P>0.05$ vs untreated control, ANOVA with 1580 Dunnett's multiple comparisons vs untreated control). w, Quantification of the lamellipodial 1581 area in vehicle- or fasudil hydrochloride-treated control and GS ${ }^{\mathrm{KD}}$ ECs (mean \pm s.e.m.; $n=6$ 1582 independent experiments; ${ }^{*} P<0.05$ and ${ }^{N S} P>0.05$ vs untreated control, ANOVA with 1583 Dunnett's multiple comparisons vs untreated control). x, Quantification of the lamellipodial 1584 area in vehicle-, ML7- or peptide 18-treated $G^{K D}$ and control ECs (mean \pm s.e.m.; $n=4$ 1585 independent experiments of which 3 experiments included the ML7-treatment; ${ }^{*} P<0.05$ vs 1586 untreated control, ANOVA with Dunnett's multiple comparisons vs untreated control). y, 1587 Scratch wound closure in vehicle-, ML7- or peptide 18-treated GS ${ }^{K D}$ and control ECs 1588 (mean \pm s.e.m.; $\mathrm{n}=3$ independent experiments; ${ }^{\star} P<0.05$ vs untreated control, ANOVA with 
1589 Dunnett's multiple comparisons vs untreated control). z, Fold-changes (vs untreated 1590 control ECs) in F-actin levels from phalloidin-stained vehicle-, ML7- or peptide 18-treated $1591 G^{K D}$ ECs (mean \pm s.e.m.; n=4 independent experiments of which 3 included the peptide 1592 18-treatment; ${ }^{*} P<0.05$ vs untreated control, one sample $t$ test). aa, Fold-changes (vs 1593 untreated control ECs) in pMLC levels from pMLC-immunostained vehicle-, ML7- or 1594 peptide 18-treated GS ${ }^{\mathrm{KD}}$ ECs (mean \pm s.e.m.; $\mathrm{n}=4$ independent experiments of which 3 1595 included the peptide 18-treatment; ${ }^{*} P<0.05$ vs untreated control, one sample $t$ test. Exact $1596 \quad P$ values: (n) MSO: 0.0372; GS $^{\mathrm{KD}}$ : 0.0060; RHOJ ${ }^{\mathrm{KD}}: 0.0051$; (u) $\mathrm{GS}^{\mathrm{KD}}$ vs ctrl: 0.0045; Fasu 1597 vs ctrl: 0.9596; GS ${ }^{\mathrm{KD}}+$ Fasu vs ctrl: 0.8857; (v) $\mathrm{GS}^{\mathrm{KD}}$ vs ctrl: 0.0199; Fasu vs ctrl: 0.8309; $1598 \mathrm{GS}^{\mathrm{KD}}+$ Fasu vs ctrl: 0.9327; (w) $\mathrm{GS}^{\mathrm{KD}}$ vs ctrl: 0.0074; Fasu vs ctrl: 0.5906; GS ${ }^{\mathrm{KD}}+$ Fasu 1599 vs ctrl: 0.9900; (x) GS ${ }^{K D}$ vs ctrl: 0.0011; $G S^{K D}+M L 7$ vs ctrl: 0.0079 ; $G S^{K D}+$ pep.18 vs ctrl: 1600 0.0017; (y) $\mathrm{GS}^{\mathrm{KD}}$ vs ctrl: 0.0034; $\mathrm{GS}^{\mathrm{KD}}+\mathrm{ML7}$ vs ctrl: 0.0022; $\mathrm{GS}^{\mathrm{KD}}+$ pep.18 vs ctrl: 1601 0.0040; (z) GS ${ }^{\mathrm{KD}}: 0.0058$; ML7: 0.0072; pep.18: 0.0888; (aa) GS ${ }^{\mathrm{KD}}$ : 0.0369; ML7: 0.0021; 1602 pep.18: 0.1672. Fasu., fasudil hydrochloride; pep. 18, peptide 18. Scale bar is $20 \mu \mathrm{m}$ in (b$1603 \mathrm{~m})$ and $100 \mu \mathrm{m}$ in (o-t). For gel source images, see Supplemental Information Fig. 1.

1604

1605 Extended Data FiguRe 7: RhOGTPASE LOCALIZATION AND INTERACTION WITH GS

1606 a, Co-IP assays showing no detectable interaction between GS and RHOA or RHOC (red 1607 asterisk indicates a non-specific band (also present in the IgG controls and unaffected by 1608 silencing of RHOA or RHOC). Picture shown is representative for 3 independent 1609 experiments. b, Co-IP of overexpressed GS and RHOJ-EGFP or $\triangle \mathrm{N}-\mathrm{RHOJ}-E G F P$ in ECs.

1610 Quantifications are mean \pm s.e.m.; $\mathrm{n}=4$ independent experiments; ${ }^{*} P<0.05$, one-sample $t$ 1611 test. In some of the experiments, the expression of $\Delta$ N20-RHOJ-EGFP was lower than 1612 expression of RHOJ-EGFP. To correct for this, densitometric quantification was performed 1613 and signals in IP lanes were normalized to input signals. c, Immunoblotting for RHOA and 1614 RHOC on cytosolic $(c)$ and membrane $(m)$ fractions of ECs with NaK as membrane marker 
and GAPDH as cytosolic marker. Picture shown is representative for 3 independent experiments. d, Bimolecular fluorescence complementation (BiFC) assay with GS coupled to the N-terminal half of EGFP, and RHOJ coupled to the C-terminal half of EGFP. Only when GS and RHOJ are in close proximity, the two EGFP half-sites complement each other and form a functional EGFP. e, Percentage of ECs displaying BiFC upon overexpression of GS-EGFP ${ }^{1 / 2}$ and RHOJ-EGFP ${ }^{2 / 2}$ or GS-EGFP ${ }^{1 / 2}$ and $\triangle \mathrm{N}-\mathrm{RHOJ}$ EGFP ${ }^{2 / 2}$. Data are mean \pm s.e.m.; $n=3$ independent experiments; * $P<0.05$; Student's $t$ test. f, Scheme for SPT-PALM imaging under TIRF illumination with the plasma membrane depicted at the top. The TIRF region is bright (whereas the part outside the TIRF region is grayed out) and contains the plasma membrane and its immediately adjacent space (not shown at exact relative dimensions). Weight and number of arrowheads represent velocity of single particles (the photoswitchable fluorescent protein (PSFP) or the PSFP coupled to the protein of interest (here GS)). The PSFP is activated upon entry into the TIRF region and is color-coded differently inside vs outside of the TIRF region. PSFP-GS displays reduced velocity in the TIRF region, presumably because of palmitoylation and membrane association of GS. g, Scheme for in-cell labeling of proteins with clickable alkynecontaining palmitoylation probes and subsequent biotin-azide clicking. $X$ represents a 1632 palmitoylated protein, $\mathrm{N}_{3}$ is the biotin-coupled azide. $\mathbf{h}-\mathbf{i}$, Rate of CoA release from 1633 palmitoyl-CoA as readout for recombinant human GS autopalmitoylation while varying either the doses of palmitoyl-CoA (h) or the amounts of recombinant GS (i) (meanıs.e.m.; $\mathrm{n}=4$ independent experiments for $\mathrm{h}$ and $\mathrm{n}=5$ for $\mathrm{i}$; ${ }^{*} P<0.05$, ANOVA with Dunnett's multiple comparisons vs $0 \mu \mathrm{M}$ palmitoyl-CoA or vs $0.5 \mu \mathrm{g}$ recombinant GS). j, Representative GS 1637 immunoblot (of 3 independent experiments) for binding of recombinant human GS to 1638 palmitoyl-CoA agarose. IF=input fraction; $\mathrm{FT}=$ flow through; W8=wash fraction 8; 1639 SDS=eluate. k-m, Representative images of RHOJ-EGFP localization in ECs under 1640 vehicle-treatment $(k)$ or treatment with 2BP (pan-palmitoylation inhibitor) (I). Red 
1641 arrowheads indicate EGFP signal at membrane ruffles, which was quantified as percent of 1642 total cellular area $(\mathrm{m})$ (mean \pm s.e.m.; $\mathrm{n}=4$ independent experiments; ${ }^{\star} P<0.05$ vs vehicle1643 treated, paired Student's $t$ test). $\mathbf{n}$-p, Representative images of ECs overexpressing wt 1644 RHOJ-EGFP (n), RHOJ-EGFP point-mutated on cysteine residue 3 (C3A) (o) or RHOJ1645 EGFP point-mutated on cysteine residue $11(\mathrm{C} 11 \mathrm{~A})(\mathrm{p})$. Red arrowheads indicate RHOJ at 1646 the plasma membrane. ECs that are not completely in the field of view have been masked 1647 out in blue. q, Quantification of the RHOJ-EGFP positive area at the plasma membrane as 1648 a percentage of total cell area. Data are mean \pm s.e.m.; $n=5$ independent experiments; $1649{ }^{*} P<0.05$; ANOVA with Dunnett's comparison vs wt RHOJ. r, RHOJ immunoblotting on 1650 membrane vs cytosolic fractions from ECs overexpressing wt RHOJ-EGFP (RHOJ WT), 1651 RHOJ-EGFP point-mutated on cysteine residue 3 (RHOJ C3A) or RHOJ-EGFP point1652 mutated on cysteine residue 11 (RHOJ C11A), with NaK as membrane marker and 1653 GAPDH and a-tubulin as cytosolic markers. s, Densitometric quantification of RHOJ/NaK 1654 as determined in (r). Data are mean \pm s.e.m.; $n=6$ independent experiments; * $P<0.05$; one 1655 sample $t$ test. t, RHOJ activity in ECs under vehicle- or 2BP-treatment (blots are 1656 representative of 3 independent experiments; densitometric quantification in arbitrary units $1657(\mathrm{AU})$ is mean \pm s.e.m; ${ }^{*} P<0.05$, paired Student's $t$ test $v s$ vehicle-treated). u, RHOJ 1658 immunoblotting for control and $\mathrm{GS}^{\mathrm{KD}}$ ECs overexpressing RHOJ (RHOJ ${ }^{\mathrm{OE}}$ ) subjected to 1659 acyl-RAC. The cleaved bound fraction (cBF) represents palmitoylated RHOJ. IF is the 1660 input fraction, whereas the cleaved unbound fraction (cUF) and the preserved bound 1661 fraction $(\mathrm{pBF})$ are controls showing depletion of RHOJ from the thioester cleaving reagent 1662 and near absence of non-specific binding of RHOJ to the resin (see Methods). 1663 Densitometric quantification of cBF/IF is shown (mean \pm s.e.m; $n=3$ independent 1664 experiments; ${ }^{*} P<0.05$, one-sample $t$ test $v s$ control). v, GRAPHICAL ABSTRACT: Left side: 1665 Autopalmitoylation allows endothelial GS to interact directly (or indirectly) with the 1666 RhoGTPase RHOJ and to sustain RHOJ's palmitoylation, membrane localization and 
activity (reflected by GTP binding). RHOJ activity then sustains normal EC migration and

1668 lamellipodia formation, and keeps actin stress fiber formation at levels, promoting normal EC migration and vessel branching in vivo. Through mechanisms that are incompletely understood, active RHOJ inhibits signaling of the RHOA/B/C - ROCK - (p)MLC pathway 1671 (itself known to promote stress fiber formation). The relative contribution of a direct effect of RHOJ on migration $v s$ the indirect effect through $\mathrm{RHOA} / \mathrm{B} / \mathrm{C}-\mathrm{ROCK}-(\mathrm{p}) \mathrm{MLC}$ remains

1673

1674

1675

1676

1677

1678

1679

1680

1681

1682

1683

1684

1685

1686

1687

1688

1689

1690

1691

1692

to be determined. Reduced opacity of RHOA/B/C, ROCK and (p)MLC indicates reduced signaling of this pathway. GTP: guanosine triphosphate. Right side: Loss of endothelial GS renders RHOJ less active (visually reflected by fewer palmitoylated, membrane-bound RHOJ proteins), and weakens the brake on the RHOA/B/C - ROCK - (p)MLC pathway. The resulting excessive stress fiber formation causes ECs to lose migratory capacity and reduces vessel branching in vivo. Dashed lines indicate reduced activity; red $\mathrm{X}$ indicates GS blockade; question mark indicates unknown mechanisms. Exact $P$ values: (b) 0.0153; (e) 0.0334; (h) 2 vs $0 \mu \mathrm{M}$ : 0.6327; 5 vs $0 \mu \mathrm{M}$ : 0.2841; 10 vs $0 \mu \mathrm{M}$ : 0.1090; 20 vs $0 \mu \mathrm{M}$ : 0.0339; 40 vs $0 \mu \mathrm{M}: 0.0034$; (i) 1 vs $0.5 \mu \mathrm{g}$ : 0.5806; 2 vs $0.5 \mu \mathrm{g}: 0.0319$; 4 vs $0.5 \mu \mathrm{g}$ :0.0037; 8 vs $0.5 \mu \mathrm{g}: 0.0001 ; 16$ vs $0.5 \mu \mathrm{g}$ : 0.0001; (m) 0.0313; (q) RHOJ C3A vs RHOJ WT: 0.0001; RHOJ C11A vs RHOJ WT: 0.0001; (s) RHOJ C3A vs RHOJ WT: 0.0015; RHOJ C11A vs RHOJ WT: 0.0007; (t) 0.0051; (u) 0.0461. Scale bar is $200 \mu \mathrm{m}$ in k,l,n-p. For gel source images, see Supplemental Information Fig. 1.

\section{Extended Data Figure 8: Possible mOleCUlAR MODEL OF GS AUtOPALMitoylation}

a. Structure of human GS and of its bifunnel-shaped catalytic site. Schematic representation of the GS decamer in top and front view with individual subunits $A$ and $B$ labeled and colored gray and green, respectively. Close-up of the bifunnel catalytic site which is formed between subunits A and B. The GS decamer has 10 active sites, each located at the interface of two adjacent subunits. ATP enters from the top whereas 
1693 glutamate enters from below; Manganese ions $\left(\mathrm{Mn}^{2+}\right)$ are shown as metalic spheres. $\mathbf{b}$.

1694 Molecular dynamics (MD) simulation of palmitoyl-CoA in the catalytic cleft of GS predicts 1695 that, while the head of palmitoyl-CoA is tightly bound to the adenine binding site, the tail 1696 can point in opposing directions with respect to the protein's principal axis. The most 1697 representative structures of the two alternative poses observed during the long MD 1698 simulations for palmitoyl-CoA binding to GS (in blue, seen from two different perspectives) 1699 are shown in red ( $A$, tail bending upwards) and green (B, tail bending downwards). c. 1700 Detailed view on the main conformation - conformation A - is shown in more details. The 1701 sulfur atom of palmitoyl-CoA (which is immediately adjacent to the carbon on which the 1702 nucleophilic attack occurs) (colored yellow) approaches the highly conserved C209 (also 1703 colored yellow), with an interatomic distance (S-S) that during the simulations reversibly 1704 fluctuates between 3 and $8 \AA$. The hydrophobic tail positions itself along grooves 1705 characterized by the presence of hydrophobic residues. Color coding: carbons are grey, 1706 nitrogens blue, phosphorous golden and oxygens red. Cysteines and serines within $5 \AA$ 1707 from the palmitoyl tail are highlighted in yellow and orange, respectively. The hydrophobic 1708 residues around the tail are shown in green. $\mathbf{d}$. Detailed view on conformation B where the 1709 tail is found in a buried hydrophobic cleft, with the sulfur at a distance of $5 \AA$ or less from 1710 the conserved serines 65 and 75 and the tail occupying the site of the GS inhibitor MSO. 1711 Details are shown of the extensive steric clash between MSO and the secondary binding 1712 pose $(B)$ observed in palmitoyl-CoA MD simulations. Palmitoyl-CoA is represented as 1713 sticks with standard atomic colours. MSO is shown in cyan and its position is taken from 1714 the 2QC8 entry in the protein databank. Cysteines and serines within $5 \AA$ from the 1715 palmitoyl tail are highlighted in yellow and orange, respectively. The hydrophobic residues 1716 around the tail are shown in green. e. GS immunoblotting after streptavidin pull-down of 1717 biotin-azide clicked lysates from 16C-YA (palmitoylation probe) labeled HEK-293T cells 1718 overexpressing wild type GS or GS point-mutated for C209. The input shows the level of 
1719 GS overexpression. Representative blot for 4 independent experiments is shown. f-g.

1720 Quantification of total sprout length (f) and number of sprouts per spheroid (g) for control 1721 and $G S^{K D}$ ECs with or without overexpression of shRNA resistant C209A-point mutated 1722 GS ( $\mathrm{rGS}^{\mathrm{C} 209 \mathrm{~A}-\mathrm{OE}}$ ) (mean \pm s.e.m.; $\mathrm{n}=4$ independent experiments; ${ }^{*} P<0.05$ vs control, 1723 ANOVA with Dunnett's multiple comparison vs control). $\mathbf{h}$. Schematic representation of 1724 protein autopalmitoylation. Upon binding of palmitoyl-CoA to the protein, free CoA (gray 1725 oval) is released and can be detected. i. Recombinant wild-type (WT) and point-mutated 1726 (R324C and R341C) GS were incubated with different doses of palmitoyl-CoA in a cell-free 1727 system at physiologcial $\mathrm{pH}$. Release of CoA per minute was determined as a direct 1728 readout for protein autopalmitoylation. Data are mean \pm s.e.m. of 3 (R324C and R341C) 1729 and 4 (WT) independent experiments. ${ }^{N S} P>0.05 ;{ }^{*} P<0.05$ according to two way ANOVA 1730 comparing the entire dose-response to the dose-response of WT GS. j. Different amounts 1731 of recombinant WT, R324C and R341C GS were incubated with a fixed amount of 1732 palmitoyl-CoA $(40 \mu \mathrm{M})$ and CoA release per minute was determined as readout for 1733 autopalmitoylation. Data are mean \pm s.e.m. of 4 (R324C and R341C) and 5 (WT) 1734 independent experiments. ${ }^{N S} P>0.05 ;{ }^{\star} P<0.05$ according to two way ANOVA comparing the 1735 entire dose-response to the dose-response of WT GS. The data for WT GS from panels (i) 1736 and (j) are also included in Extended Data Fig. 7 as stand-alone data, but are included 1737 here too for comparison purposes. k. Boyden chamber migration for control, GS ${ }^{\mathrm{KD}}, \mathrm{GS}^{\mathrm{KD}}+$ $1738 \mathrm{rGS}^{\mathrm{OE}}(\mathrm{r}=$ shRNA-resistant; $\mathrm{OE}=$ overexpression $), \mathrm{GS}^{\mathrm{KD}}+\mathrm{rGS}^{\mathrm{R} 341 \mathrm{C}-\mathrm{OE}}$ and $\mathrm{GS}^{\mathrm{KD}}+$ $1739 \mathrm{rGS}^{\mathrm{R} 324 \mathrm{C}-\mathrm{OE}} \mathrm{ECs}$, all under mitomycin C-treatment (mean \pm s.e.m.; $\mathrm{n}=3$ independent 1740 experiments; ${ }^{N S} P>0.05 ;{ }^{*} P<0.05$, ANOVA with Dunnett's multiple comparison vs control). 1741 Exact $P$ values: (f) $G S^{K D}$ vs ctrl: 0.0004; $\mathrm{GS}^{\mathrm{KD}}+\mathrm{rGS}^{\mathrm{C} 209 \mathrm{~A}-\mathrm{OE}}$ vs ctrl: 0.0004; (g) $\mathrm{GS}^{\mathrm{KD}}$ vs 1742 ctrl: 0.0001; GS ${ }^{\mathrm{KD}}+\mathrm{rGS}^{\mathrm{C} 209 \mathrm{~A}-\mathrm{OE}}$ vs ctrl: 0.0001; (i) R324C vs WT: 0.8228; R341C vs WT: 1743 0.7530; (j) R324C vs WT: 0.1331; R341C vs WT: 0.0003; (k) GS ${ }^{\mathrm{KD}}$ vs ctrl: 0.0054; GS ${ }^{\mathrm{KD}}+$ 
$1744 \mathrm{rGS}^{\mathrm{OE}}$ vs ctrl: $0.8152 ; \mathrm{GS}^{\mathrm{KD}}+\mathrm{rGS}^{\mathrm{R} 341 \mathrm{C}-\mathrm{OE}}$ vs ctrl: $0.3645 ; \mathrm{GS}^{\mathrm{KD}}+\mathrm{rGS}^{\mathrm{R} 324 \mathrm{C}-\mathrm{OE}}$ vs ctrl:

1745 0.2118. For gel source images, see Supplemental Information Fig. 1.

1746

1747

1748

1749

1750

1751

1752

1753

1754 


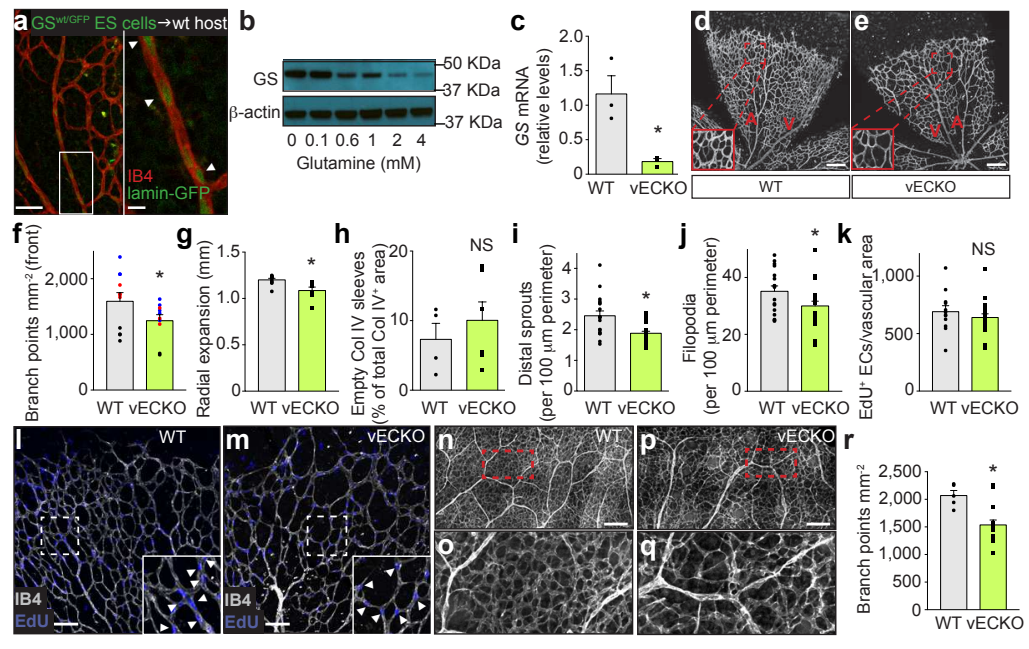




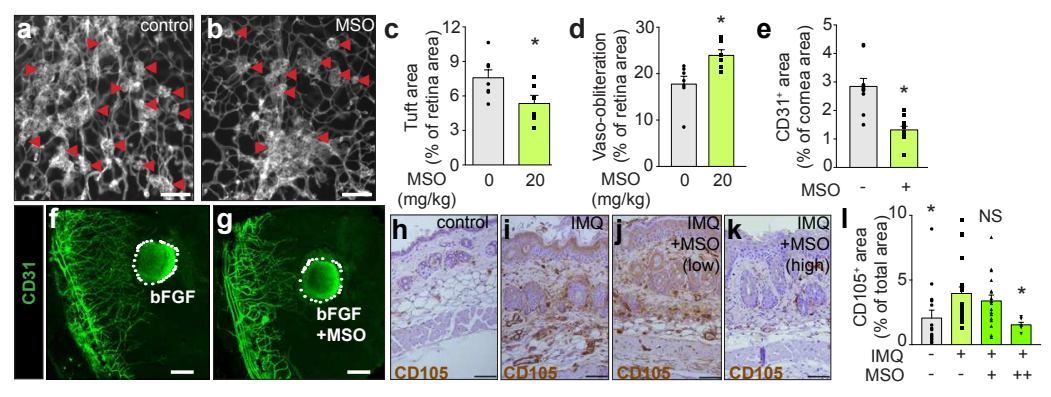



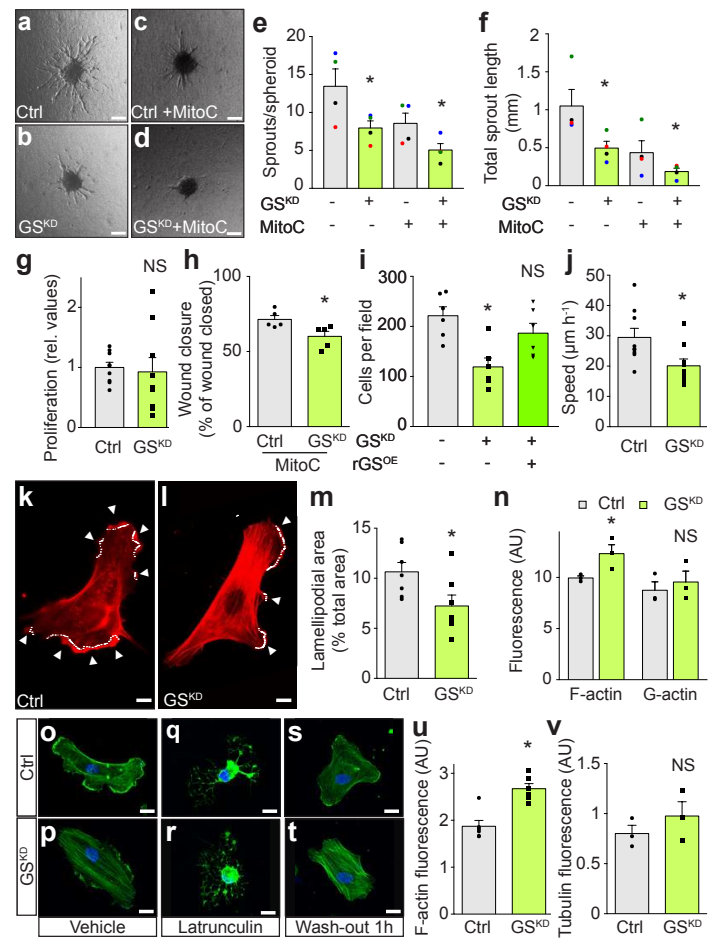

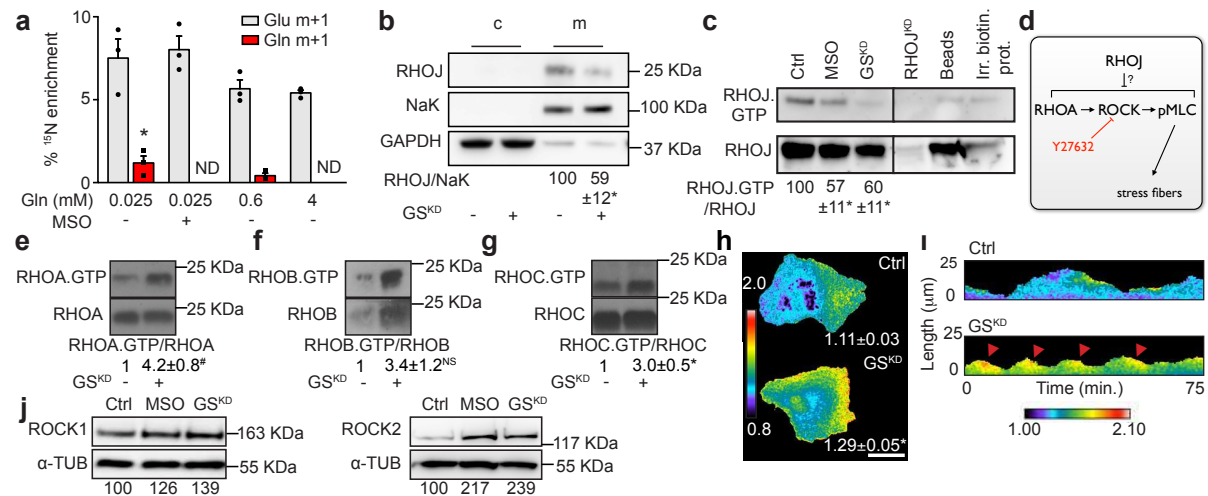

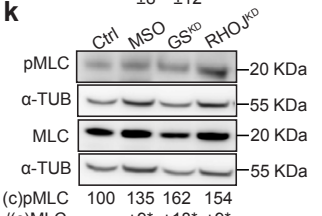

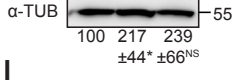

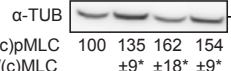
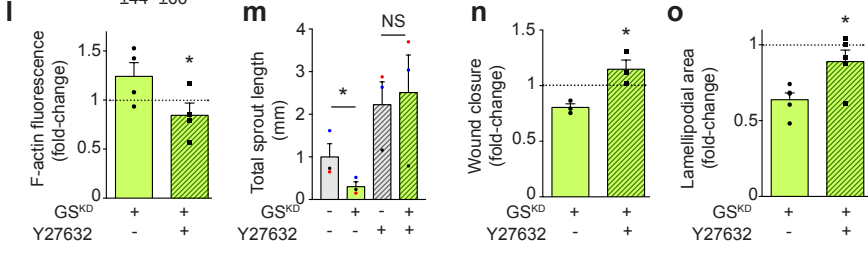


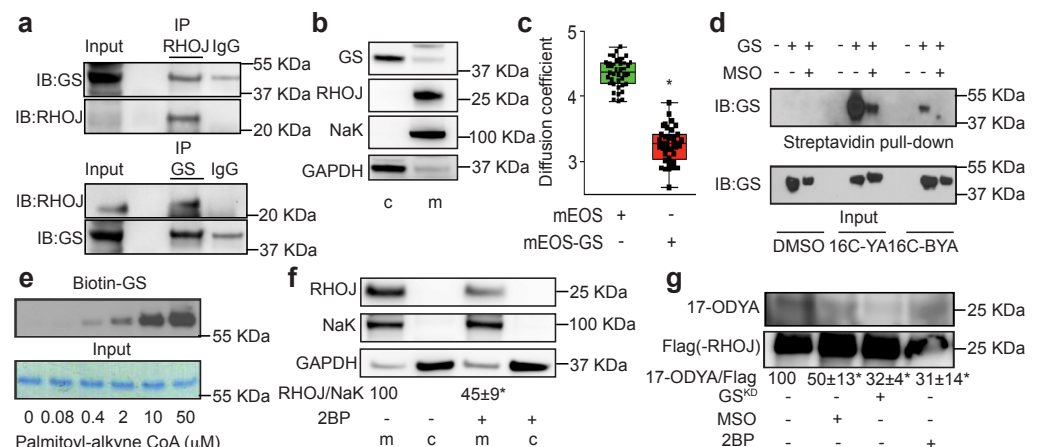

